\title{
The effect of the geometric features of the turbulent/non-turbulent interface on the entrainment of a passive scalar into a jet $\oplus^{\oplus}$
}

Cite as: Phys. Fluids 32, 095114 (2020); https://doi.org/10.1063/5.0019860

Submitted: 25 June 2020 . Accepted: 25 August 2020 . Published Online: 15 September 2020

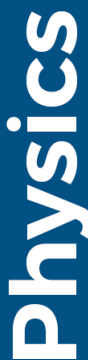

(D) Khashayar F. Kohan, and (D) Susan Gaskin

\section{COLLECTIONS}

EP This paper was selected as an Editor's Pick
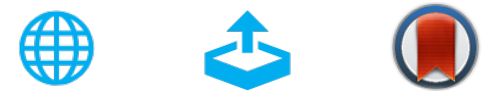

\section{ARTICLES YOU MAY BE INTERESTED IN}

Statistical analysis of deformation of a shock wave propagating in a local turbulent region Physics of Fluids 32, 096107 (2020); https://doi.org/10.1063/5.0019784

Turbulent/non-turbulent interfaces detected in DNS of incompressible turbulent boundary layers

Physics of Fluids 30, 035102 (2018); https://doi.org/10.1063/1.5022423

Mechanisms of entrainment in a turbulent boundary layer

Physics of Fluids 33, 035105 (2021); https://doi.org/10.1063/5.0040575

Physics of Fluids SPECIAL TOPIC: Tribute to Frank M. White on his 88th Anniversary 


\title{
The effect of the geometric features of the turbulent/non-turbulent interface on the entrainment of a passive scalar into a jet ()
}

\author{
Cite as: Phys. Fluids 32, 095114 (2020); doi: 10.1063/5.0019860 \\ Submitted: 25 June 2020 - Accepted: 25 August 2020 • \\ Published Online: 15 September 2020

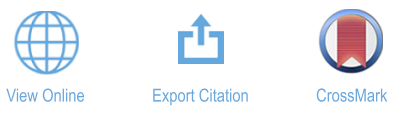

Khashayar F. Kohan (D) and Susan Gaskin ${ }^{\text {a) }}$ (D)

\section{AFFILIATIONS}

Department of Civil Engineering and Applied Mechanics, McGill University, Montréal, Québec H3A OC3, Canada

a) Author to whom correspondence should be addressed:susan.gaskin@mcgill.ca

\begin{abstract}
We consider the scalar concentration field in the proximity of the turbulent/non-turbulent interface (TNTI) of a round momentum-driven turbulent jet at $R e=10600$. Orthogonal cross sections of the jet are taken at 50 nozzle diameters from the nozzle exit using planar laser-induced fluorescence. The conditional scalar concentration is evaluated along the interface-normal direction, identifying the thickness of the TNTI region as $0.64 \lambda$ (where $\lambda$ is the Taylor microscale). Conditioning the scalar concentration within the TNTI revealed higher values of the passive scalar in the vicinity of the boundary elements shaped by large vorticity structures, i.e., isosurface points with low curvature (flat regions), small interface angle, and large radial distance from the jet centerline. In contrast, small vorticity structures near the boundary manifesting with high interface curvature, high interface angle, and small radial distance are associated with lower concentration values. Using the current experimental resolution, we find that high concentrations near the far boundary points persist up to a distance of $0.40 \lambda-0.48 \lambda$ into the TNTI region, after which boundary points closer to the jet centerline exhibit larger concentration values along the interface-normal direction, similar to the fully turbulent region. The cross correlation analysis showed that in regions characterized by low streamwise momentum, there are positive, albeit small, scalar correlations between the non-turbulent and the TNTI regions. The latter may imply local detrainment of the fluid particles containing the scalar at far radial positions.
\end{abstract}

Published under license by AIP Publishing. https://doi.org/10.1063/5.0019860

\section{INTRODUCTION}

It is understood that the sharp and highly convoluted turbulent/non-turbulent interface (TNTI) present at the outermost boundary of free turbulent flows controls the transport of mass, momentum, and scalars (e.g., heat) between the turbulent and the non-turbulent (irrotational) fluid. For example, in non-premixed combustion systems, combustion occurs at a low stoichiometric mixture fraction, defined as the mass fraction of the fuel stream. Low fuel mixture fractions are generally found at the outer edge of the turbulent flow, i.e., within the TNTI. Therefore, the reaction rate is greatly affected by the geometry of the interfacial layer. ${ }^{1}$ The flow properties are governed by sharp gradients across the thickness of the TNTI, when evaluating their conditional average relative to the position of the boundary. ${ }^{2-5}$ The TNTI is a composite layer consisting of two sublayers: a viscous superlayer (VSL), ${ }^{6-8}$ where viscous diffusion dominates the evolution of the vorticity field, and a turbulent sublayer (TSL), ${ }^{9,10}$ dominated by inviscid processes.

The TNTI geometry has been the subject of several experimental and numerical studies in recent years. It has been shown that the thickness of the TNTI is comparable to the radius of the large vorticity structures (LVSs) populating the outer boundary of free turbulent flows, ${ }^{11}$ which scales with the Taylor microscale, $\lambda$. However, at high Reynolds numbers, $R e_{\lambda} \gtrsim 200$, the thickness of the TNTI begins to scale with the Kolmogorov microscale, $\eta=\left(v^{3} / \varepsilon\right)^{1 / 4}$, due to the fragmented and less coherent shape of the LVSs close to the TNTI. ${ }^{12}$ It has been observed that the probability density function (PDF) of the TNTI position is nearly Gaussian in different turbulent flows such 
as jets, ${ }^{13}$ wakes, ${ }^{2}$ mixing layers, ${ }^{14}$ and boundary layers. ${ }^{15}$ In addition, the PDF of the TNTI curvature reveals that the boundary is dominated by smooth bulges with radii of curvature of the order of the integral length scale and $\lambda$ in shear-free turbulent fronts ${ }^{16}$ and other free turbulent flows, ${ }^{17-22}$ respectively.

Another line of active research has been the local entrainment velocity at the TNTI, $v_{n}$, which is defined as the relative velocity between the surface propagation velocity, $\boldsymbol{u}_{\boldsymbol{s}}$, and the local fluid velocity at the TNTI, $\boldsymbol{u}_{I}$, i.e., $v_{n} \hat{\boldsymbol{n}}=\boldsymbol{u}_{\boldsymbol{s}}-\boldsymbol{u}_{\boldsymbol{I}}$, where $\hat{\boldsymbol{n}}$ is the local interface-normal unit vector. Previous studies in turbulent jets showed that local entrainment processes mostly occur through highly curved convex surface elements, defined looking from the turbulent region. ${ }^{21,23,24}$ In contrast, flat and highly curved concave TNTI points are associated with the most intense entrainment events in wakes ${ }^{18}$ and mixing layers, ${ }^{19}$ respectively. Additionally, a strong correlation between $v_{n}$ and the orientation of the TNTI relative to the mean flow direction has been observed, e.g., entrainment happens mostly across cross-streamwise and leading edges, while the interface propagates toward the turbulent region at the trailing edge. ${ }^{21,25}$ The large scale aspects of the TNTI such as its position, imposed by the largest eddies from the turbulent region, ${ }^{26,27}$ also affect the local entrainment velocity. For example, more negative values of $v_{n}$ (entrainment) are associated with interface points closer to the jet centerline. ${ }^{21}$

Historically, the entrainment process was associated with the large vorticity structures deep inside the turbulent core. These structures envelope large packets of ambient fluid, drawing them into the turbulent region at the inward cusps of the TNTI, a process known as inviscid engulfment. ${ }^{28,29}$ However, recent experimental and numerical studies revealed that the amount of irrotational fluid entrained into the turbulent flow due to the small scale molecular processes in the proximity of the TNTI, i.e., nibbling, exceeds that of the engulfment mechanism. ${ }^{3,13,30-32}$ The latter finding attests to the original idea of Corrsin and Kistler, ${ }^{6}$ who noted that the entrainment is brought about by the diffusive mechanisms across the contorted TNTI. Nevertheless, the local diffusion of the TNTI into the irrotational region is decorrelated with the local small scale turbulence. ${ }^{16,21}$ It is, in fact, the largest vorticity structures that control the entrainment rate, ${ }^{26}$ mainly through two mechanisms. The first one is inducing the irrotational entrainment wind to draw ambient fluid toward the turbulent boundary. ${ }^{33}$ The second mechanism is increasing the turbulent surface area by continuous convolution of the TNTI across the full range of scales, along which small scale diffusion acts. ${ }^{2,19,34}$ This points out the multi-scale nature of the turbulent entrainment process $^{26}$ as described by the power-law behavior of the TNTI surface area with a 3D fractal dimension of $D_{3}=2.3-2.4$, or $D_{2}=1.3-$ 1.4 in two-dimensional cuts (e.g., Refs. 15, 22, and 35-40). Moreover, the entrainment rate in pure turbulent jets (i.e., without buoyancy) only depends on turbulence production, ${ }^{41}$ a mechanism controlled by the straining rate of the largest eddy structures in the turbulent flow. $^{42}$

It is crucial to note that the local entrainment velocity of the scalar TNTI, $v_{n}^{\phi}$, is generally different from the propagation rate of the vorticity TNTI, $v_{n}$. The scalar TNTI resides inside its vorticity counterpart for $S c>1$ due to the weak diffusive nature of the scalar, ${ }^{43,44}$ and therefore, $v_{n}^{\phi}<v_{n}$. In contrast, when $S c$ is low, the scalar isosurface propagates faster and is located outside the vorticity TNTI. For scalars with $S c \approx 1$, the two TNTIs closely coincide with only slight deviations. ${ }^{14,45}$

The evolution and characteristics of a passive scalar field with low/moderate $S c$ near the TNTI have received attention previously. For example, regions of high scalar gradient are generally correlated with flat surface elements, independent of the sign of the curvature. ${ }^{46}$ When conditioning with respect to the TNTI orientation, Watanabe et al. ${ }^{25}$ found the smallest conditionally averaged concentration gradient near the trailing edge surfaces. Consistently, the scalar dissipation is shown to be much smaller near the trailing edge as compared to that near the leading edge surfaces. ${ }^{47}$ In their direct numerical simulations (DNS) of incompressible mixing layers with $0.25 \leqslant S c$ $\leqslant 8$, Watanabe et al. ${ }^{43}$ showed a large difference in the scalar value between the VSL and the TSL, due to the different origins of the fluid particles in these sublayers. The latter leads to a peak in the conditionally averaged dissipation profile between the VSL and the TSL, independent of $S c$. Furthermore, it has been shown that $S c,{ }^{44,48}$ as well as compressibility and heat release, ${ }^{49}$ can alter the dynamics of the scalar mixing and topology of the scalar TNTI.

The main goal of the present study is to analyze the geometry of the TNTI in orthogonal cross sections of a turbulent round jet using planar laser-induced fluorescence (PLIF). The sharply contorted TNTI is identified by applying a threshold to the scalar concentration field having a large Schmidt number $(S c=2000)$. We then proceed to obtain the geometric features of the outer boundary of the interfacial layer. The geometry of the interface is characterized by its curvature, $\kappa_{I}$, the cosine of the angle between its normal and radial unit vectors, $\cos \left(\psi_{I}\right)$, and its radial position from the jet centerline, $r_{I}$. These parameters will be defined in Sec. III. To complete the assessment of the interface geometry, the power-law behavior of the TNTI is investigated using the box-filtering method. We also examine the relationship between the value of the passive scalar at the TNTI and the geometry of the interface. The latter contributes to a better understanding of the scale of the vortical structures responsible for the mixing of passive scalars, immediately after entrainment of the fluid particles into the turbulent region. Finally, using the conditionally averaged cross correlation profiles, we attempt to explain the effect of the interface geometry on the local entrainment/detrainment of the passive scalar. Accurate predictions of the scalar behavior in the vicinity of the TNTI are important in many engineering and environmental applications.

This article is organized as follows. Section II describes the experimental facility, the data acquisition procedure, and the conditional averaging method with respect to the TNTI position. In Sec. III, we present the main results and discuss the relationship between the TNTI geometry and the evolution of the passive scalar field in its vicinity. Finally, in Sec. IV, a summary of our findings and conclusions are presented.

\section{EXPERIMENTAL METHOD}

\section{A. Apparatus}

The experiments were carried out in a $1.7 \times 1.5 \times 0.9 \mathrm{~m}^{3}$ opentop glass tank with walls of tempered glass to provide optical access for the PLIF imaging. The tank was filled with water, and sufficient time was allowed for the water to come to rest before the 
experiment. The jet was issued from a copper tube with an inner diameter of $d=8 \mathrm{~mm}$, which extends vertically for $1.6 \mathrm{~m}$ and after a $90^{\circ}$ bend extends horizontally for an additional $0.12 \mathrm{~m}$ ( 15 jet diameters), thus ensuring a fully developed turbulent flow at the nozzle exit. The dyed fluid was pumped to a constant-head reservoir positioned at $\sim 3 \mathrm{~m}$ above the nozzle exit. The purpose of the constanthead was to feed the jet while maintaining a constant pressure difference with the supply reservoir. An outlet located just below the inlet of the constant-head served to direct the excess dyed water back to the supply to ensure a steady level in the head reservoir. The flow rate was maintained at the desired level $\left(4 \mathrm{l} \mathrm{min}^{-1}\right)$ using a flow meter (Omega FL50002A) with a ball valve to set the flow rate and a solenoid valve to turn the jet on and off. The constant flow rate resulted in a turbulent round jet with $R e=U_{e} d / v=10600$, where $U_{e}$ and $v$ are the nozzle-exit velocity and kinematic viscosity, respectively. This Reynolds number is sufficient for the scalar field to reach a well-mixed state. ${ }^{50} \mathrm{~A}$ schematic of the experimental facility is shown in Fig. 1.

The laser beam used for the PLIF experiments originated from a continuous argon-ion laser (Coherent Innova 90) operating in the single-line mode at a wavelength of $514.5 \mathrm{~nm}$ and an output power of $1 \mathrm{~W}$. The passive scalar was disodium fluorescein with a high Schmidt number of $S c=2000$ to minimize the effect of molecular diffusion and to prevent blurring of the images. Planar cross sections of the jet were illuminated by a laser-sheet of $2 \mathrm{~mm}$ thickness created by a laser scanning device, consisting of a focusing lens and an eight-sided polygonal rotating mirror (Lincoln Laser Co.). The rotating mirror spun at a maximum rate of $12000 \mathrm{rpm}$ and scanned the laser beam across the measurement area, producing a laser-sheet with relatively uniform density. The beam was directed into the laser-sheet-forming optics using two mirrors (see Fig. 2).

The images were taken using a $2016 \times 2016$ pixel $^{2}$, 12-bit monochromatic CCD camera (pco.dimax) with an object lens of $55 \mathrm{~mm}$. The fluorescent light emitted from the dye first reached an optical filter (ThorLabs FGL550) attached to the lens, which rejected the scattered light of the laser beam and only transmitted the fluorescent signal. The sensitivity of the camera was increased by means of

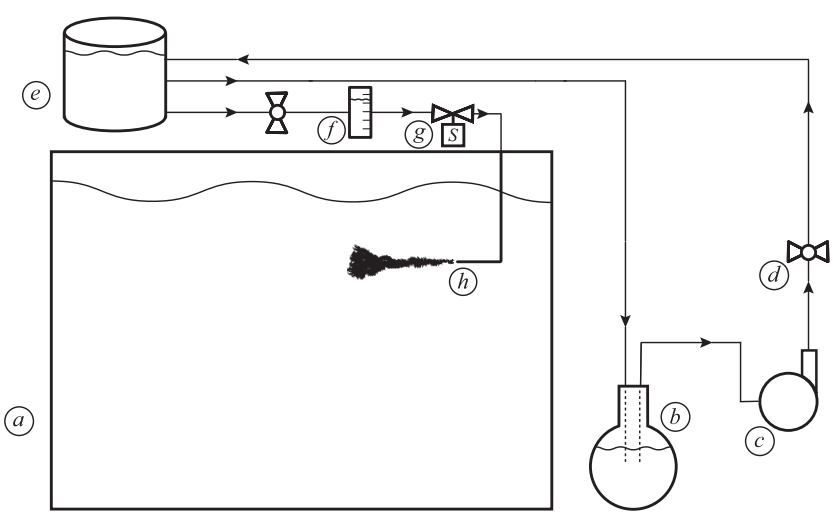

FIG. 1. Schematic of the experimental apparatus including the (a) tank, (b) dyedfluid supply reservoir, (c) pump, (d) ball valve, (e) constant-head reservoir, (f) flow meter, (g) solenoid valve, and ( $h$ ) jet nozzle. The figure is not to scale (side view).
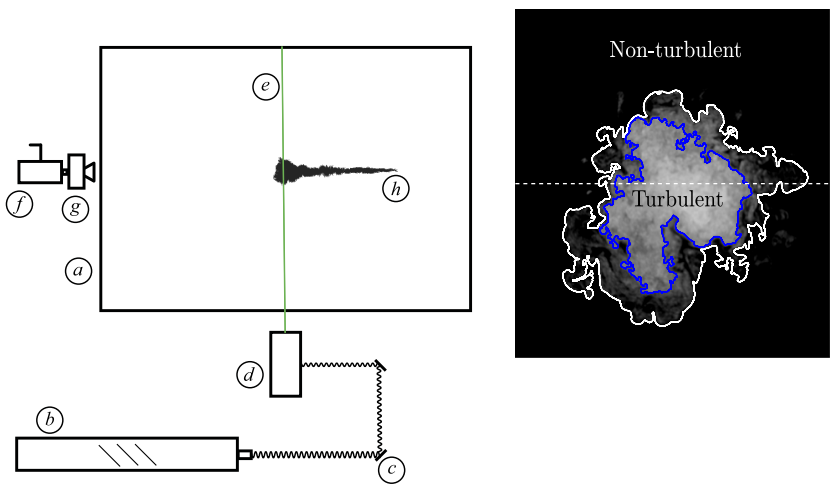

FIG. 2. Schematic of the PLIF system consisting of the (a) tank, (b) laser, (c) mirror, (d) laser scanning device, (e) laser-sheet, (f) CCD camera, (g) intensifier, and (h) jet nozzle. The figure is not to scale (top view). The instantaneous scalar concentration field is represented in logarithmic scaling, with the TNTI and half-width (where $\phi / \phi_{c}=0.5$ ) isocontours shown by white and blue lines. The dashed line intersects with the centerline and demarcates the upper and lower FOVs.

an image intensifier (Video Scope V34-1854) mounted on the camera. The intensifier reduced the size of the usable field of view (FOV) to a 1600 pixel diameter circle in the middle of the original FOV. The coupling of the image intensifier and the camera rendered the effective central region more sensitive than the edges of the FOV, making a pixel-by-pixel calibration necessary. An instantaneous PLIF image is also shown in Fig. 2. As can be seen, the instantaneous interface is highly contorted. The streamwise, transverse, and spanwise coordinates are denoted $x, y$, and $z$, respectively. The radial coordinate and azimuthal angle are represented by $r$ and $\theta$. The reader is referred to Refs. 51 and 52 for additional details on the experimental facility.

\section{B. Data acquisition and flow characterization}

Measurements for this study were performed in the far-field at a downstream distance of $x / d=50$ from the nozzle. The image was focused such that each pixel corresponded to an area of $250 \times 250 \mu \mathrm{m}^{2}$ in the object field. At this resolution, the complete orthogonal spatial extent of the jet was captured at the measurement location. The data were recorded at a rate of $30 \mathrm{~Hz}$ with $30 \mathrm{~ms}$ exposure. The total duration of the experiment was about $44 \mathrm{~s}$, during which a total of 1315 instantaneous scalar fields were recorded. A pixel-by-pixel calibration was performed to convert the intensity data, taken from the camera, to actual concentration values for each scalar image. The calibration process consisted of seven successive PLIF tests for homogeneous mixtures of known (and increasing) dye concentration in a small container $(1 \times 0.5$ $\times 0.5 \mathrm{~m}^{3}$ ), placed in the measurement location. Prior to the conversion, the background intensity was determined without adding any dye to the jet, and this was subtracted from each scalar intensity field, i.e.,

$$
\frac{\phi(x=\text { const; } y, z)}{\phi_{\text {calib }}}=\frac{I(y, z)-\overline{I_{\text {back }}}(y, z)}{\overline{I_{\text {calib }}}(y, z)-\overline{I_{\text {back }}}(y, z)},
$$


TABLE I. Experimental parameters in the present study. The Taylor Reynolds number is defined as $R e_{\lambda}={\overline{u^{\prime 2}}}^{1 / 2} \lambda / v$. The parameters $U_{c},{\overline{u^{\prime 2}}}^{1 / 2}, b_{\phi, 1 / 2}$, and $\eta_{B}$ denote the centerline velocity, rms velocity, concentration half-width, and Batchelor microscale, respectively. Note that here $\eta=\left(v^{3} / \varepsilon\right)^{1 / 4}, \lambda={\overline{u^{\prime 2}}}^{1 / 2} \sqrt{15 v / \varepsilon}$, and $\eta_{B}=\eta / \sqrt{S c}$. All these quantities are measured at the jet centerline at a downstream distance of $x / d=50$.

\begin{tabular}{ccccccccccc}
$R e$ & $R e_{\lambda}$ & $U_{c}\left(\mathrm{~cm} \mathrm{~s}^{-1}\right)$ & ${\overline{u^{\prime 2}}}^{1 / 2}\left(\mathrm{~cm} \mathrm{~s}^{-1}\right)$ & $b_{\phi, 1 / 2}(\mathrm{~mm})$ & $\varepsilon\left(\mathrm{cm}^{2} \mathrm{~s}^{-3}\right)$ & $\eta(\mathrm{mm})$ & $\lambda(\mathrm{mm})$ & $\eta_{B}(\mu \mathrm{m})$ & $\begin{array}{c}\text { (PLIF pixel spacing) } \\
(\mathrm{mm})\end{array}$ & $\begin{array}{c}\text { No. of scalar } \\
\text { fields }\end{array}$ \\
\hline 10600 & 140 & $17.0^{\mathrm{a}}$ & $4.59^{\mathrm{a}}$ & 48.5 & $32.6^{\mathrm{a}}$ & 0.13 & 3.11 & 2.9 & $0.25(=2 \eta)$ & 1315 \\
\hline \hline
\end{tabular}

${ }^{\mathrm{a}}$ The velocity data are taken from the study of Khorsandi.

where $\phi_{\text {calib }}$ is a uniform concentration field used for the pixel-bypixel calibration and $\overline{I_{\text {calib }}}(y, z)$ is its corresponding ensemble average intensity. The ensemble average of the background intensity due to dark noise is denoted $\overline{I_{\text {back }}}(y, z)$. The validity of Eq. (1) depends on the negligible attenuation of the laser light and linearity of the fluorescent signal with concentration, which are both confirmed for the present study.

All the flow properties are obtained for the upper half of the FOV, as shown in Fig. 2. The isocontours of the TNTI and the concentration half-width encircle the jet centerline. The procedure for determining the isocontours will be described in detail in Sec. II C. The value of the concentration half-width, $b_{\phi, 1 / 2}$, is calculated by averaging the radial distance between the half-width points and the centerline for all 1315 scalar concentration fields. The concentration half-width value is reported in Table I and is used to normalize the measured lengths in this study. The resolution of the passive scalar field at high $S c$ should be assessed relative to the Batchelor microscale, $\eta_{B}$. As can be seen in Table I, the current experimental facilities do not resolve the smallest length scales of the scalar concentration, and we are not able to fully capture the passive scalar gradient. However, for high-Sc flows, as discussed in Sec. II C and the Appendix, a PLIF resolution close to several times the Kolmogorov microscale, $\eta$, should be adequate to obtain the conditionally averaged concentration and root mean square (rms) concentration profiles relative to the interface position in the outer regions of the flow. The experimental parameters and measured lengths at the jet centerline are summarized in Table I. Note that the parameters regarding the velocity field are estimated from a previous study, ${ }^{51}$ undertaken in the same laboratory with a comparable $R e$.

Before proceeding to the conditional statistics and results, validation tests are performed, showing that the jet is representative of a fully developed turbulent flow. Figures 3(a) and 3(b) show the radial distributions of mean and rms scalar concentrations, normalized by the ensemble averaged centerline concentration value, $\phi_{c}$, and centerline rms value, ${\overline{\phi^{\prime 2}}}_{c}^{1 / 2}$, respectively. The scalar value at a given $(r, \theta)$ is approximated using bilinear interpolation. Subsequently, the mean and rms concentrations at a given radial position are calculated as the azimuthally averaged values at the radius $r$. In general, the profiles show good agreement with previous experiments carried out in round jets, ${ }^{52-55}$ given the differences in the initial conditions, Reynolds numbers, and employed techniques (e.g., PLIF, smoke scattering, Raman scattering). Figure 3(c) presents the PDF of the passive scalar at the centerline, normalized by $\phi_{c}$. The PDF roughly follows a Gaussian distribution, shown by the orange line, which is in good agreement with the data of Papanicolau and List. ${ }^{56}$ The zero probability of finding pure unmixed fluid $(\phi \approx 0)$ at the centerline implies that the scalar concentration field is fully mixed deep inside the turbulent core. In summary, the results of Fig. 3 follow the

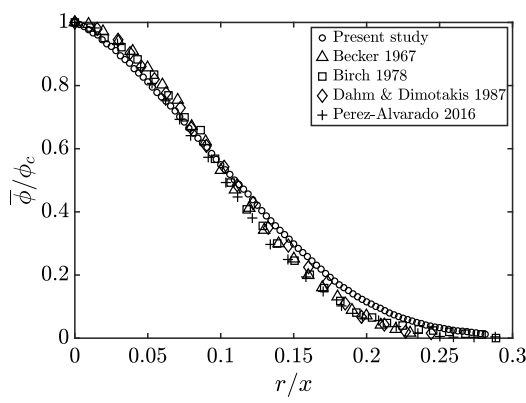

(a)

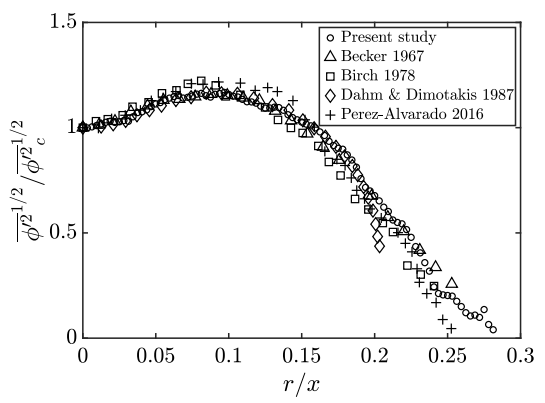

(b)

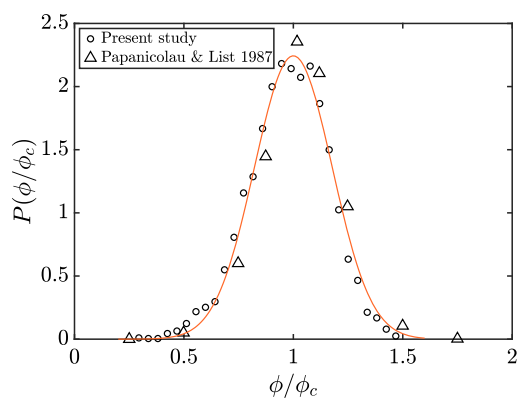

(c)

FIG. 3. Large scale concentration statistics at $x / d=50$, normalized by the centerline concentration and rms values. The radial position from the jet centerline, $r$, is normalized by the downstream distance from the nozzle exit, $x$. (a) Mean and (b) rms profiles of scalar concentration are compared with the experimental results from the studies of Becker et al., ${ }^{53}$ Birch et al., ${ }^{54}$ Dahm and Dimotakis, ${ }^{55}$ and Perez-Alvarado. ${ }^{52}$ (c) PDF of the scalar concentration at the jet centerline, $r / x=0$, compared with the profile reported by Papanicolau and List. ${ }^{56}$ In addition, shown with the orange line is the Gaussian fit to the present PDF. 
accepted behavior of a passive scalar reported in turbulent round jets and, therefore, serve to validate the current study.

\section{Conditional statistics}

As mentioned earlier in the Introduction, the vorticity TNTI is a composite layer made up of two sublayers: the viscous superlayer (VSL) and the turbulent sublayer (TSL). ${ }^{9}$ Analogously, the scalar TNTI is comprised of two sublayers, albeit with different characteristics at different $S c .{ }^{44}$ For example, for high-Sc flows such as in the present study, Silva and da Silva ${ }^{44}$ proposed a viscous-convective superlayer that closely follows the VSL in space and an inertialconvective sublayer with a much smaller thickness compared to that of the TSL. Nevertheless, for both TNTIs, these two sublayers are bounded on one side by the outer boundary of the interfacial region, which shall be called the TNTI outline hereafter, and by the start of the turbulent core region on the other side. The TNTI outline is often detected empirically by applying a low-magnitude threshold to a scalar field, e.g., enstrophy, ${ }^{2,17}$ passive scalar, ${ }^{13,37}$ or turbulent kinetic energy. ${ }^{15}$ Indeed, the TNTI outline defined using the passive scalar generally does not coincide with that found with the other scalar fields, ${ }^{43,44,48}$ except for $S c \approx 1 .{ }^{14,45}$ Here, we detect the TNTI outline by applying a threshold to the scalar concentration field following the procedure described in Refs. 37 and 58. We proceed by introducing a conditional concentration, defined as the pixel averaged concentration value across the FOV where the local concentration exceeds the given threshold, $\phi>\phi_{t}$,

$$
\tilde{\phi}=\frac{\sum(\phi n)_{\mid \phi>\phi_{t}}}{\sum n_{\mid \phi>\phi_{t}}},
$$

where $n$ denotes the number of pixels. The threshold is then selected as the concentration value where $\tilde{\phi}$ exhibits a sharp change in its derivative, i.e., inflection point. Similar to that reported by Mistry et al., ${ }^{37}$ islands of turbulent jet fluid (points where scalar concentration exceeds the threshold but are detached from the main scalar field) have been excluded from the conditional averaging, while holes (points inside the jet region with a concentration less than the threshold value) are included. The conditional concentration and its derivative are shown in Fig. 4. As the concentration threshold, $\phi_{t}$, approaches the mean centerline value, the detected turbulent region breaks into several disconnected patches. Therefore, it becomes progressively harder to distinguish between the main jet fluid and the turbulent islands. However, the scalar concentration threshold is usually much lower than $\phi_{c}$, which allows us to identify the detrained patches rather easily and remove them from the analysis. The derivative of the conditional concentration, $\mathrm{d} \tilde{\phi} / \mathrm{d} \phi_{t}$, exhibits sharp changes at two thresholds, i.e., $\phi_{t} / \phi_{c}=0.05$ and $\phi_{t} / \phi_{c}=0.13$ [see Fig. 4(b)]. The higher threshold is applied to each instantaneous scalar concentration field to detect the turbulent region. The lower threshold represents the average concentration value of the ambient (background) fluid and cannot sharply differentiate between the turbulent and non-turbulent regions, as made evident by the visual inspection of the instantaneous TNTI outlines after applying $\phi_{t} / \phi_{c}=0.05$. This phenomenon was also observed in the bimodal histograms of the scalar intensity ${ }^{58}$ and vorticity, ${ }^{32}$ where the higher threshold with the lower peak was selected to identify the interface. The TNTI outline is then selected as the longest continuous

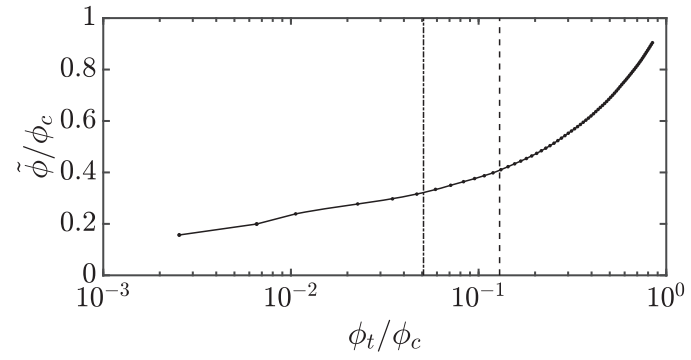

(a)

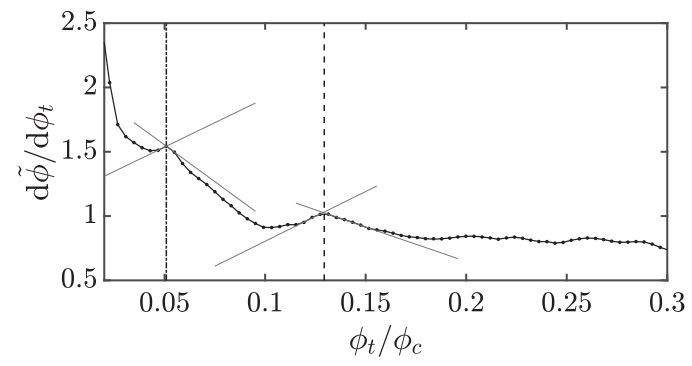

(b)

FIG. 4. (a) Conditional scalar concentration, $\tilde{\phi}$, as defined in Eq. (2). (b) Derivative of the conditional concentration, $\mathrm{d} \tilde{\phi} / \mathrm{d} \phi_{t}$, computed using a compact sixth-order scheme ${ }^{57}$ The conditional and derivative profiles are presented with semi-log and linear scaling, respectively.

isocontour along $\phi / \phi_{c}=0.13$, with the turbulent islands and nonturbulent holes (as identified by the absence of the scalar) excluded. Averaging the results over the 1315 instantaneous fields revealed that the detached turbulent islands constitute $3.81 \%$ of the turbulent jet fluid, while the contribution of the non-turbulent holes amounts to $1.41 \%$. The half-width isocontour can also be identified using a similar procedure.

The conditionally averaged profiles (denoted \langle\rangle$_{I}$ ), relative to the position of the interface, are evaluated in a local coordinate system normal to the TNTI outline, $x_{n}$. The unit normal of the interface is defined as $\hat{\boldsymbol{n}}=(\nabla \phi /|\nabla \phi|)_{I}$, where the subscript $I$ denotes points along the TNTI outline. Using this definition, the positive directions of $\hat{\boldsymbol{n}}$ and $x_{n}$ point toward the turbulent core region [see Fig. 5(a)]. In order to increase the accuracy, the spatial derivatives are calculated by a sixth-order finite-difference compact scheme. ${ }^{57}$ The flow properties are interpolated onto $x_{n}$ using a bilinear interpolation method. In some cases, the status of the flow (turbulent or non-turbulent) changes along the interface-normal coordinates more than once, i.e., the interpolated points may cross the TNTI outline, irrotational holes, or detached turbulent islands at points other than their first crossing at the origin of $x_{n}$. These points, as well as those remaining along the $x_{n}$ direction, are discarded and not included in the conditional averaging procedure. Flow quantities are interpolated up to a distance of one concentration half-width from the TNTI outline on both sides to ensure that all interpolated points are within the FOV. Moreover, $b_{\phi, 1 / 2}$ is sufficiently large so that the interpolated points in the turbulent region pass the transitional adjustment layer. ${ }^{13}$ Figures 5(b)-5(d) present several conditionally averaged profiles. 


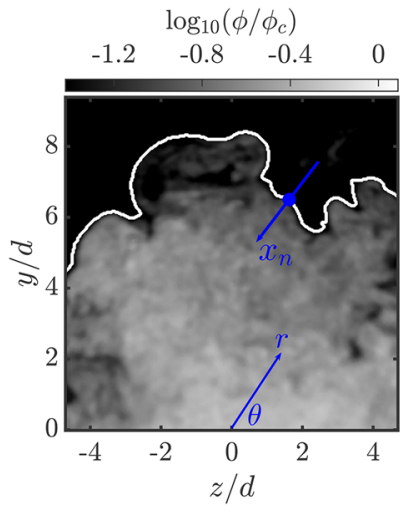

(a)

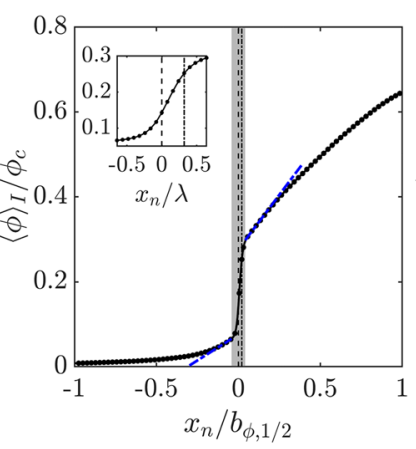

(b)

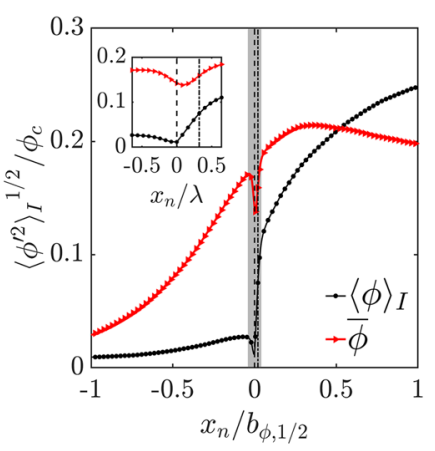

(c)

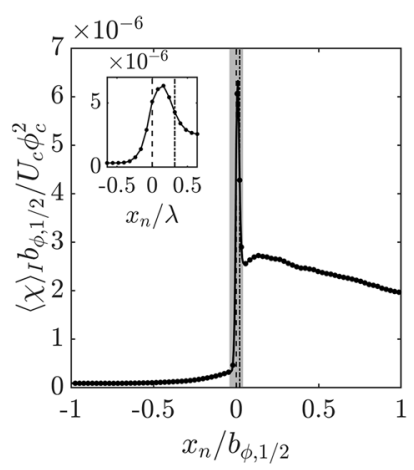

(d)

FIG. 5. (a) Part of an instantaneous scalar concentration field normalized by the mean centerline concentration at $x / d=50$, presented with logarithmic scaling. The jet centerline is located at $(y, z)=(0,0)$, with the radial position from the centerline calculated as $r=\sqrt{y^{2}+z^{2}}$. In addition, shown is an example of the local coordinate system $x_{n}$ used to compute the conditional statistics. The TNTI outline is the white isocontour along $\phi / \phi_{c}=0.13$. Conditionally averaged profiles of (b) scalar concentration, (c) scalar concentration rms, and (d) scalar concentration dissipation. The black circles (red triangles) in (c) denote the conditional scalar rms defined relative to the conditional (conventional) mean concentration. The mean centerline concentration, $\phi_{c}$, centerline velocity, $U_{c}$, and scalar half-width, $b_{\phi, 1 / 2}$, are used for non-dimensionalization. The narrow region, where the conditional concentration jump occurs, is shown by the gray bar. The turbulent region is defined as $x_{n}>0$, while the non-turbulent region is $x_{n}<0$. The inset plots in (b)-(d) zoom in the gray bar with their $x$-axis normalized by the Taylor microscale, $\lambda$. The dashed and dashed-dotted lines represent $x_{n} / \lambda=0($ TNTI outline) and $x_{n} / \lambda=0.32$ (investigation point), respectively.

Previously, Watanabe et al. ${ }^{43}$ investigated the dependence of the conditional mean scalar profile on $S c$. Their simulations revealed that for high $S c$, the molecular diffusion has a negligible effect on the averaged scalar concentration evolution across the TNTI, and that the Kolmogorov microscale is a more relevant length scale at the TNTI as compared to the Batchelor scale. Therefore, capturing the conditionally averaged concentration profile does not require spatial resolution down to $\eta_{B}$, and the current PLIF resolution $(\approx 2 \eta)$ should be adequate for this purpose. The effect of the spatial resolution on the conditionally averaged profiles is further discussed in the Appendix. The mean conditional concentration profile is depicted in Fig. 5(b). The TNTI thickness is calculated as the span, starting from the TNTI outline, across which the jump in the scalar concentration occurs. Similar to that reported by Eisma et al., ${ }^{59}$ the interfacial layer is identified as the region with a sharp scalar gradient located between the linear fits (shown by blue dashed lines) to the conditional concentration profile in both the turbulent and non-turbulent regions. Using this method, we find the TNTI thickness to be $\sim 0.64 \lambda$, which is in good agreement with the previous results observed in different turbulent flows with comparable $R e$ (e.g., see Refs. 2, 11, 13, 25, 43, and 60). Furthermore, the ratio between the TNTI thickness and $\eta$ is $\sim 15$ [or $\mathscr{O}(10)$ ], consistent with the constant scaling of the TNTI thickness and the Kolmogorov microscale. ${ }^{12}$ The sudden increase in the concentration value could also be observed in Fig. 2, as the isocontour of the scalar half-width was close to that of the TNTI outline. Two differently defined conditionally averaged scalar rms profiles are shown in Fig. 5(c). Their difference lies in the average reference value from which we are measuring the fluctuations, i.e., $\left\langle\phi^{\prime 2}\right\rangle_{I}^{1 / 2}=\left\langle\left(\phi-\langle\phi\rangle_{I}\right)^{2}\right\rangle_{I}^{1 / 2}$ is defined relative to the conditional mean, while $\left\langle\phi^{\prime 2}\right\rangle_{I}^{1 / 2}=\left\langle(\phi-\bar{\phi})^{2}\right\rangle_{I}^{1 / 2}$ is defined relative to the conventional mean, $\bar{\phi}$. The conditionally averaged rms defined with respect to the conventional mean is larger in the non-turbulent region but smaller in the turbulent region. The conventional (or classical) averaging smears out flow quantities across the TNTI thickness. It is for this reason that the conditional profile defined relative to $\bar{\phi}$ experiences a more gradual transition from the non-turbulent region to the turbulent region. Nonetheless, both profiles are governed by sharp jumps across the interfacial layer with a local minimum within the TNTI region. The presence of the local minimum at or near $x_{n}=0$ could be attributed to the predefined nature of the TNTI outline, limiting the scalar fluctuations at the boundary. Finally, the conditionally averaged profile of scalar dissipation is provided in Fig. 5(d). The scalar dissipation is defined as

$$
\chi=2 D \frac{\partial \phi}{\partial x_{i}} \frac{\partial \phi}{\partial x_{i}}
$$

where $D$ is the molecular diffusivity. As expected, the conditional concentration dissipation exhibits a narrow and strong peak within the scalar TNTI, followed by a rather constant value in the turbulent region. The region between the TNTI outline and the $\langle\chi\rangle_{I}$ peak has a thickness of $0.16 \lambda$ and is characterized by highly inhomogeneous scalar mixing. This region could be perceived as analogous to the VSL of the vorticity TNTI independent of $S c$, 43,44 where inhomogeneity is maximal. ${ }^{4,5}$ Therefore, an intermediate point between the dissipation peak and the inner boundary of the TNTI region $\left(x_{n} / \lambda=0.64\right)$ is chosen in Sec. III B to study the scalar field within the interfacial layer, e.g., at $x_{n} / \lambda=0.32$, as shown by a dashed-dotted line in Figs. 5(b)-5(d). We note that resolution comparable to the Batchelor microscale is required to capture the true scalar concentration gradient in the vicinity of the TNTI outline. However, as shown in the Appendix, the location of the dissipation peak remains approximately unaltered for decreasing spatial resolutions, and the sharp 


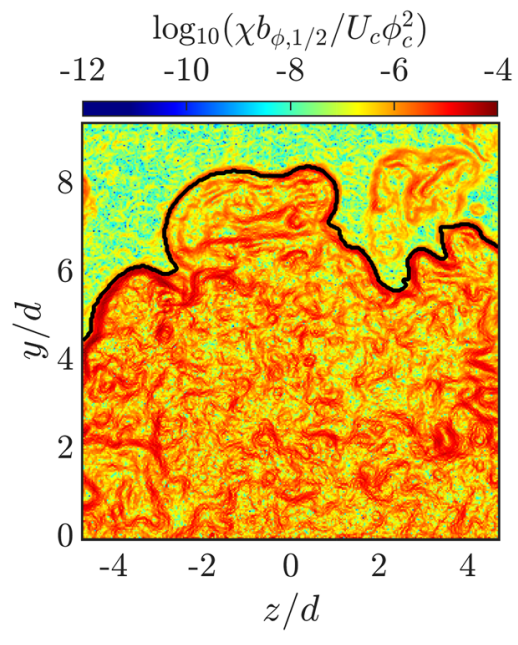

FIG. 6. Contour of the passive scalar dissipation field, $\chi$, for the same region as in Fig. 5(a), presented with logarithmic scaling. The TNTI outline is shown by the thick black line.

jump, albeit underestimated, can be captured. Similar profiles of $\langle\chi\rangle_{I}$ can also be found in previous studies. $14,44,48,61$

The scalar dissipation field provides a more robust criterion to detect the TNTI outline than the passive scalar itself. ${ }^{14,44}$ However, $\chi$ is more susceptible to numerical errors and, hence, is harder to measure than $\phi$. Figure 6 shows the scalar dissipation rate for the same region as in Fig. 5(a). As can be seen, large values of $\chi$ are concentrated in sheet-like structures, ${ }^{62}$ which are present near the TNTI outline and also inside the turbulent core. The former is due to the sudden transition from the non-turbulent to the turbulent region [also see Fig. 5(d)], while the latter is attributed to the internal intermittency (or ramp-cliff structures) of the passive scalar field. ${ }^{63}$ Large values of $\chi$ can be seen in the top-right corner of Fig. 6, which are apparently detached from the main turbulent jet. The same detached island is also observed for the scalar concentration field in Fig. 5(a). Our experimental data are limited to a two-dimensional description of the flow, and without the volumetric information, whether the detached regions are completely disconnected from the jet fluid or are attached to the turbulent region in a different streamwise plane cannot be determined. Nonetheless, as mentioned earlier, these 2D disconnected regions add up to only $3.81 \%$ of the total jet area and will not significantly affect the results. Since the turbulent and nonturbulent regions are demarcated similarly by $\chi$ and $\phi$ contours, we conclude that the present criterion for detecting the TNTI outline is valid.

\section{RESULTS}

\section{A. Geometric features of the TNTI outline}

The evolution of the enstrophy and passive scalar fields has been shown to be strongly correlated with the TNTI geometry. ${ }^{20,25,46}$ In addition, the interface geometry has not yet been experimentally investigated for the orthogonal cross section of a high-Re turbulent jet, as previous studies used streamwise and $3 \mathrm{D}$ cuts of the flow. It is beneficial to first analyze the geometric characteristics of the TNTI outline and then condition the value of the scalar concentration on these properties. Specifically, we turn our attention to the radial position of the TNTI outline, $r_{I}$, its curvature, $\kappa_{I}$, and the cosine of the interface angle, $\cos \left(\psi_{I}\right)$ (see Fig. 7). The radial position of the interface is the Euclidean distance between the TNTI outline and the jet centerline. The present TNTI outline is a planar curve, and therefore, its curvature value can be evaluated using the expression

$$
\kappa_{I}=\left(\frac{\frac{\mathrm{d} y}{\mathrm{~d} s} \frac{\mathrm{d}^{2} z}{\mathrm{~d} s^{2}}-\frac{\mathrm{d} z}{\mathrm{~d} s} \frac{\mathrm{d}^{2} y}{\mathrm{~d} s^{2}}}{\left(\left(\frac{\mathrm{d} y}{\mathrm{~d} s}\right)^{2}+\left(\frac{\mathrm{d} z}{\mathrm{~d} s}\right)^{2}\right)^{3 / 2}}\right)_{I},
$$

where $\frac{\mathrm{d}}{\mathrm{d} s}$ denotes the derivative operator with respect to the TNTI outline length and is calculated as

$$
\frac{\mathrm{d}}{\mathrm{d} s}=\frac{1}{|\nabla \phi|}\left(\frac{\partial \phi}{\partial y} \frac{\partial}{\partial z}-\frac{\partial \phi}{\partial z} \frac{\partial}{\partial y}\right) .
$$

Using the current definition of $\hat{\boldsymbol{n}}$, convex (ridges) and concave (bulges) surface elements are identified as $\kappa_{I}>0$ and $\kappa_{I}<0$, respectively. The deviation of the interface unit normal from the inward radial unit vector along the TNTI outline, $\hat{r}$, is quantified by the cosine of the angle between them,

$$
\cos \left(\psi_{I}\right)=\hat{\boldsymbol{r}} \cdot \hat{\boldsymbol{n}} .
$$

The TNTI outline tends to fold back on itself as $\cos \left(\psi_{I}\right) \rightarrow-1$, i.e., whenever $\hat{\boldsymbol{r}}$ oppositely aligns with $\hat{\boldsymbol{n}}$. Examples of different values of $\cos \left(\psi_{I}\right)$ are provided in Fig. 7 .

Figure 8 shows the PDF of the TNTI outline geometric features. Similar to that reported by previous studies in round jets, ${ }^{13,21}$ the PDF of $r_{I}$ normalized by the scalar half-width follows a Gaussian distribution [Fig. 8(a)]. The average value, standard deviation,

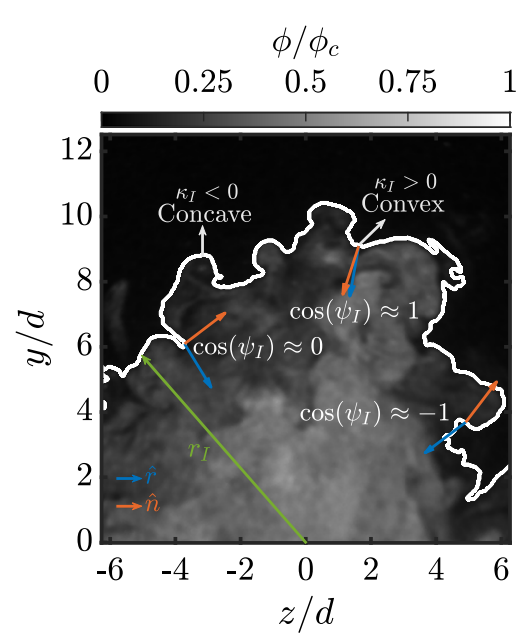

FIG. 7. Geometric features of the TNTI outline. The instantaneous scalar field normalized by the average centerline value, is presented with linear scaling. The radial and normal unit vectors along the TNTI outline are shown by the blue and orange arrows, respectively. The TNTI outline is shown by the thick white line. 


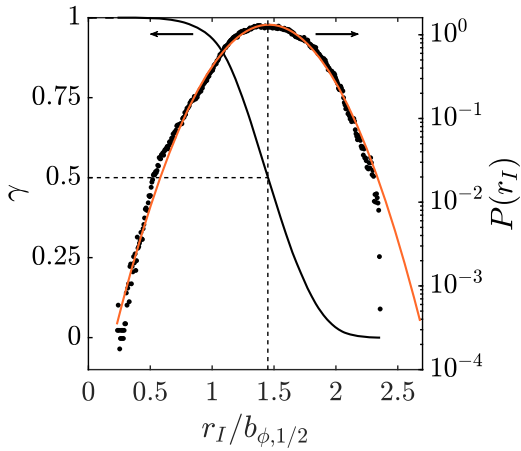

(a)

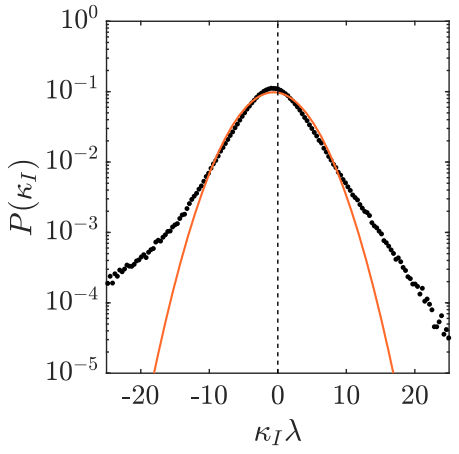

(b)

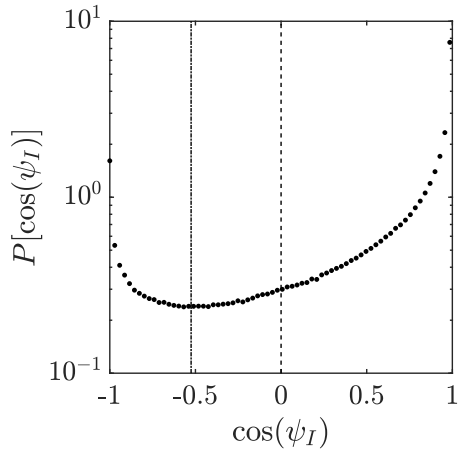

(c)

FIG. 8. PDFs of the (a) radial position of the TNTI outline, $r_{l}$, (b) interface curvature, $\kappa_{l}$, and (c) cosine of the angle between the normal and radial unit vectors of the interface, $\cos \left(\psi_{l}\right)$. The mean intermittency $\gamma$ and average radial position $\overline{r_{I}}=1.46 b_{\phi, 1 / 2}$ are also shown in (a) by the black thick line and dashed line, respectively. The Gaussian fit is shown in orange. The PDFs are all presented with logarithmic scaling.

and skewness of $P\left(r_{I}\right)$ are $\overline{r_{I}}=1.46 b_{\phi, 1 / 2},{\overline{r_{I}^{\prime 2}}}^{1 / 2}=0.30 b_{\phi, 1 / 2}$, and $\overline{r_{I}^{\prime 3}} /{\overline{r_{I}^{\prime 2}}}^{3 / 2}=-0.09$, respectively. The negative skewness of the distribution is unlike those found in a number of studies in free shear flows. ${ }^{5,13,14}$ However, those studies considered the interface envelope by neglecting the inward corrugations of the TNTI outline, which resulted in a positively skewed $P\left(r_{I}\right)$. Indeed, negative skewness has been observed when considering the actual convoluted TNTI outline. ${ }^{25}$ Figure 8 (a) also presents the mean intermittency factor, $\gamma$, calculated from the cumulative distribution of $r_{I},{ }^{6}$ i.e., $\gamma\left(r_{I}\right)=1-\int_{-\infty}^{r_{I}} P\left(r_{I}\right) \mathrm{d} r_{I}$ for a fixed downstream location. It can be seen that $\gamma$ assumes a value of 0.5 at the average radial position. The length of the TNTI outline $\left(L_{I}\right)$ is also calculated (not shown here) for each instantaneous scalar field. The cumulative average of $L_{I}$ converges to $12 b_{\phi, 1 / 2}$. The interface length ranges from $6 b_{\phi, 1 / 2}$ to $20 b_{\phi, 1 / 2}$, which is an imprint of the large scale structures deforming the TNTI outline. ${ }^{2}$

The curvature measures the rate of change in the tangent vector along the TNTI outline. It can also provide insight into the size of the eddy structures that form the TNTI; the lower the curvature value, the larger the size of the vorticity structure. As noted in Refs. 21 and 46, planar visualizations are only capable of capturing one of the principal curvatures $\left(\kappa_{1}\right.$ or $\kappa_{2}$, where $\left.\kappa_{1} \geqslant \kappa_{2}\right)$, depending on the orientation of the intersecting plane. It is known that the 3D interface of turbulent jets is skewed toward saddle-convex surface elements $\left(K_{g}=\kappa_{1} \kappa_{2}<0\right)$ with a positive mean curvature $\left(2 K_{m}=\kappa_{1}+\kappa_{2}\right.$ $>0){ }^{20,23,24}$ Therefore, the smaller principal curvature should be skewed toward negative values. In the present study, the intersecting plane produces orthogonal cross sections of the jet, where the resulting curvature, $\kappa_{I}$, is mostly formed by the lateral shear. The PDF of $\kappa_{I}$ normalized by the Taylor microscale is presented in Fig. 8(b), showing a non-Gaussian distribution. ${ }^{21,22}$ The negative skewness of the distribution implies that the component of the curvature that we capture is in fact mostly the smaller principal curvature, $\kappa_{2}$. The peak of the PDF is located at $\kappa_{I} \lambda \approx-0.8$, indicating a predominance of concave elements at the TNTI outline. The radii of the vorticity structures forming the TNTI outline can be approximated as
$R \approx 0.9 \lambda$, where $R^{-1}=\sqrt{2 K_{m}^{2}-K_{g}}$ is the curvedness. ${ }^{18,19}$ However, as also noted in Ref. 22 , the 2D studies are unable to recover the value of the Gaussian curvature, $K_{g}$. Therefore, we evaluate the curvedness solely by substituting the mean curvature value in its expression, which results in an overestimation of the radius of the vorticity structures. Nonetheless, the current two-dimensional analysis confirms that $\lambda$-sized vorticity structures dominate the TNTI region of a round turbulent jet, similar to that found in previous studies. $11,18,19,22$

The interface angle, $\psi_{I}$, is associated with the convolution of the TNTI outline. The time-averaged cross section of a round jet is a circle, where the radial and normal unit vectors are perfectly aligned, and thus, $\cos \left(\psi_{I}\right)=1$ everywhere along the curve. The PDF of $P\left[\cos \left(\psi_{I}\right)\right]$ can be appreciated in Fig. 8(c), showing that $\hat{\boldsymbol{r}}$ and $\hat{\boldsymbol{n}}$ are preferentially aligned $\left[\cos \left(\psi_{I}\right)= \pm 1\right]$. The higher peak, as expected, is located at $\cos \left(\psi_{I}\right)=1$, while the lower peak at $\cos \left(\psi_{I}\right)=-1$ indicates the intense corrugation of the TNTI outline. The PDF also presents a minimum at $\cos \left(\psi_{I}\right) \approx-0.52$, which corresponds to an interface angle of $\psi_{I} \approx 120^{\circ}$ (shown by the black vertical line). Points along the TNTI outline with $\cos \left(\psi_{I}\right) \approx-1$ are radially folded back on themselves and are mostly formed by smaller vorticity structures (see Fig. 7).

The instantaneous interface is highly contorted across a wide range of scales as exhibited by its large scale indentations and small scale wrinkles (Fig. 2). The multi-scale nature of the TNTI outline and the entrainment process are best described using the power-law (fractal) behavior of the interface. In order to investigate the fractal property of the TNTI outline, each instantaneous scalar field is spatially filtered using a $2 \mathrm{D}$ box-filter according to $\hat{\phi}(\boldsymbol{x})=\int \phi\left(\boldsymbol{x}-\boldsymbol{x}^{\prime}\right) G\left(\boldsymbol{x}^{\prime}\right) \mathrm{d}^{2} \boldsymbol{x}^{\prime}$, where $G$ and $\hat{\phi}$ denote the boxaveraging filter kernel of width $\Delta_{f}$ and the low-pass filtered concentration field, respectively. We then apply the same threshold $\phi_{t} / \phi_{c}=0.13$ to each smoothed field and find the length of the TNTI outline. The logarithmic plot of the ensemble averaged interface length as a function of the filter width is shown in Fig. 9. In addition, shown is the local slope of the filtered TNTI outline length, $-\mathrm{d}\left[\log \left(\overline{L_{I}} / b_{\phi, 1 / 2}\right)\right] / \mathrm{d}\left[\log \left(\Delta_{f} / \lambda\right)\right]$, where we find an 


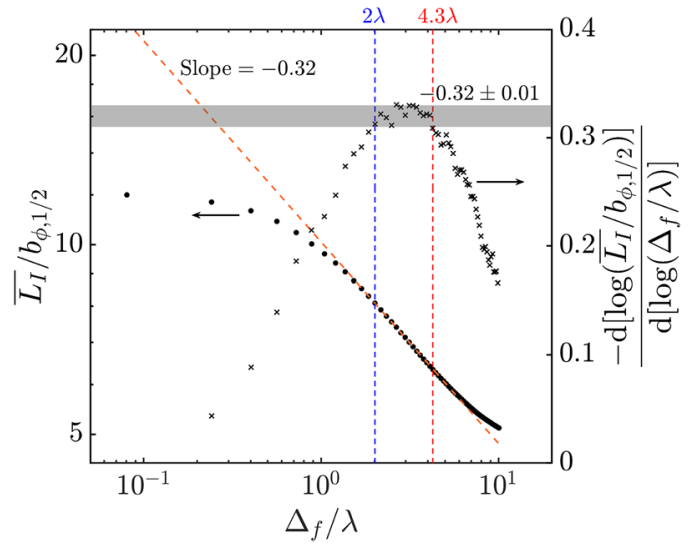

FIG. 9. Fractal scaling of the TNTI outline length using spatial filtering. The fractal dimension is calculated from the plateau of the local slope (gray bar). The vertical dashed lines delimit the filter widths between which the local slope has a value of $-0.32 \pm 0.01$

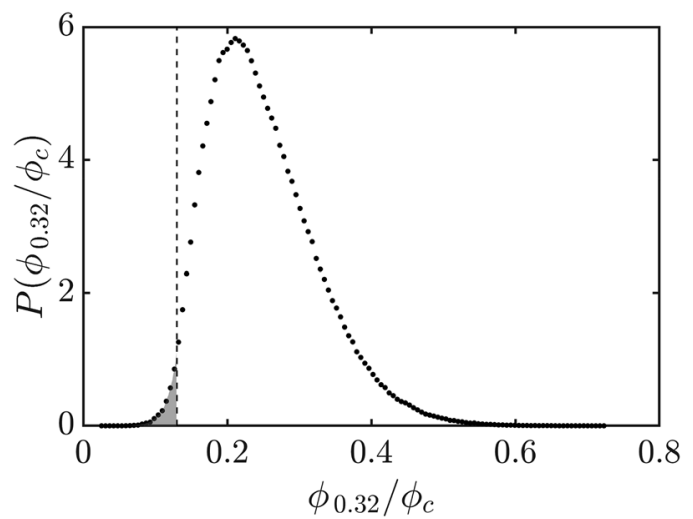

FIG. 10. PDF of the scalar concentration value at $x_{n} / \lambda=0.32$. The non-turbulent part of the distribution is shown by the gray fill. The dashed line shows the passive scalar threshold, $\phi_{0.32} / \phi_{c}=0.13$. approximate plateau of $-0.32 \pm 0.01$ between the filter widths of sizes $\Delta_{f}=2 \lambda$ and $\Delta_{f}=4.3 \lambda$. According to Mandelbrot, ${ }^{64}$ this results in a $2 \mathrm{D}$ fractal dimension of $D_{2}=1.32 \pm 0.01$, which resides well inside the accepted range of $D_{2}=1.3-1.4$, as previously found by recent studies.

\section{B. TNTI concentration conditioned on the interface geometry}

Knowing the statistical behavior of the TNTI outline geometric features, we now investigate the relationship between the scalar concentration value close to the TNTI outline (at $x_{n} / \lambda=0.32$, denoted $\left.\phi_{0.32}\right)$ and $r_{I}, \kappa_{I}$, and $\cos \left(\psi_{I}\right)$. Before proceeding further, all the interpolated flow points at $x_{n} / \lambda=0.32$ that have a non-turbulent status, as well as their corresponding TNTI outline points, are removed from the analysis. The PDF of the scalar concentration at $x_{n} / \lambda=0.32$ is presented in Fig. 10 with its irrotational contribution, $\phi_{0.32} / \phi_{c}<0.13$, shown by the gray fill. These non-turbulent points either crossed the TNTI outline into the ambient region or are within an irrotational hole. In any case, the removed points only amount to $1.38 \%$ of all the realizations and therefore are expected to have a negligible effect on the presented results. As can be seen, $\phi_{0.32}$ can be as low as $0.03 \phi_{c}$ and can reach a maximum value of $0.72 \phi_{c}$.

Figure 11 presents the joint probability density functions (JPDFs) of $\phi_{0.32}$ and the geometric features of the TNTI outline. In particular, we compare the ensemble average of the scalar concentration, $\overline{\phi_{0.32}}$, with its conditional average, $\overline{\phi_{0.32 \mid}}$, by investigating their relative difference, $e=\left[\overline{\phi_{0.32 \mid f}}-\overline{\phi_{0.32}}\right] / \overline{\phi_{0.32}}$. Here, $f$ represents $r_{I}, \kappa_{I}$, and $\cos \left(\psi_{I}\right)$ in each case. The conditional average of the scalar concentration is defined as

$$
\overline{\phi_{0.32 \mid f}}=\int \phi_{0.32} P\left(\phi_{0.32} \mid f\right) \mathrm{d} \phi_{0.32},
$$

where $P\left(\phi_{0.32} \mid f\right)$ denotes the PDF of $\phi_{0.32}$ conditioned on the independent variable $f$. This method allows us to identify the favorable conditions for accumulation of the passive scalar at the TNTI, realizing that $\phi_{0.32}$ is enhanced for $e>0$. A similar approach was employed by Mistry et al., ${ }^{21}$ who studied the influence of the TNTI outline geometry on the local entrainment velocity.

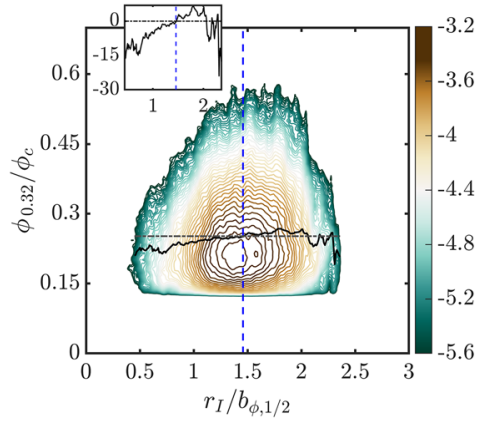

(a)

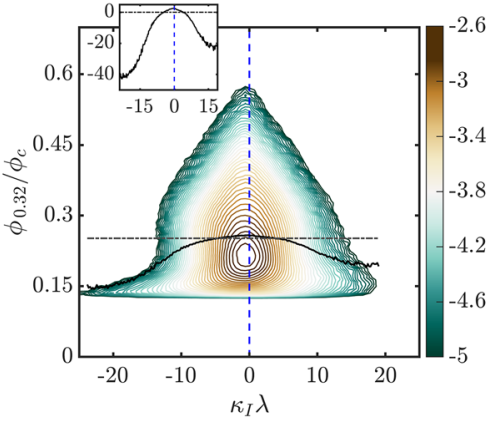

(b)

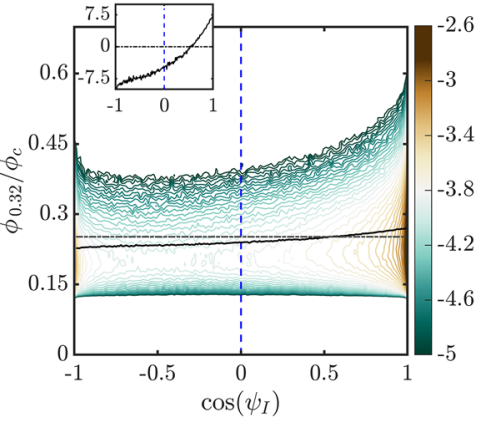

(c)

FIG. 11. JPDFs of $\phi_{0.32}$ and (a) radial position of the TNTI outline, $r_{l}$, (b) interface curvature, $\kappa_{l}$, and (c) cosine of the interface angle, $\cos \left(\psi_{l}\right)$. The ensemble average of the scalar concentration, $\overline{\phi_{0.32}}$, is shown by the gray dashed-dotted line, while its conditional average, $\overline{\phi_{0.32 \mid f}}$, is shown by the black thick line. The inset plots represent the relative difference (in percentage), e, between the conditional and the ensemble average of $\phi_{0.32}$ in each case. The JPDFs are represented with logarithmic scaling. 
We begin the analysis by considering the dependence of the scalar concentration value at $x_{n} / \lambda=0.32$ on the radial position of the TNTI outline [Fig. 11(a)]. There is clearly a positive correlation between $\phi_{0.32}$ and $r_{I}$; the value of $\overline{\phi_{0.32 \mid r_{I}}}$ exceeds that of $\overline{\phi_{0.32}}$ for radial positions larger than the mean value, i.e., $r_{I}>1.46 b_{\phi, 1 / 2}$. However, a discrepancy in this trend is observed for the furthest radial positions, beginning with the sudden plunge in the value of $\overline{\phi_{0.32 \mid r_{I}}}$ after $r_{I} \gtrsim 2 b_{\phi, 1 / 2}$. This phenomenon can be explained by the limited life cycle of the vortical structures at the boundary of the turbulent jet. Once these remote structures dissipate due to the lack of shear, the scalar transport is diffusion limited. The contribution of molecular diffusion to the total scalar transport at the edges of the free shear flows is very small at high $S c .{ }^{43}$ Without a strong local velocity field, the flow near the boundary cannot mix with the turbulent core region, and therefore, the concentration value at $x_{n} / \lambda=0.32$ remains close to the diffused non-turbulent value.

Figure 11(b) shows the correlation between $\phi_{0.32}$ and the curvature of the TNTI outline. Similar to the work of Dopazo et al., ${ }^{46}$ on average, there is a higher scalar concentration close to the interface for flatter regions of the TNTI outline. More specifically, there is a higher level of the passive scalar in the region $-4.6<\kappa_{I} \lambda<3.8$, while the remaining points along the TNTI outline with a larger absolute curvature are negatively contributing to $\overline{\phi_{0.32}}$. The conditional concentration reaches its maximum value at $\kappa_{I} \lambda=-0.2$ and is only $2.6 \%$ greater than its ensemble average, while $\overline{\phi_{0.32 \mid \kappa_{I}}}$ can decrease by up to $40 \%$ for large negative curvatures. This observation is consistent with the findings of Fig. 8(b), showing a predominance of flat surface elements formed by the large structures. Furthermore, the turbulent flow in the vicinity of the highly curved convex (positive curvature) surface elements is associated with more intense values of concentration compared to the flow near the highly curved concave (negative curvature) shaped surfaces. This can be seen in the higher tail of $\overline{\phi_{0.32 \mid \kappa_{I}}}$ for $\kappa_{I}>0$ compared with $\kappa_{I}<0$.
Figure 11(c) depicts the relationship between the value of $\phi_{0.32}$ and the cosine of the interface angle. On average, there is an increase in the value of the passive scalar near the TNTI outline where $\hat{r}$ preferentially aligns with $\hat{\boldsymbol{n}}$. Particularly, the conditional average of the scalar concentration is intensified when $\cos \left(\psi_{I}\right) \geqslant 0.5$. On the other hand, $\phi_{0.32}$ is minimized as $\cos \left(\psi_{I}\right) \rightarrow-1$, i.e., the TNTI region is characterized by lower concentration values when small vorticity structures are present (see Sec. III A).

Summarizing the results of Fig. 11, there is an accumulation of the passive scalar near the points along the TNTI outline with moderate to large radial positions from the jet centerline. The concentration value is also enhanced in the proximity of low curvature surface elements, independent of the curvature sign. The positive alignment of the radial and normal unit vectors of the TNTI outline also results in greater values of scalar concentration. These scenarios (large $r_{I}$, small $\kappa_{I}$, and $\cos \left(\psi_{I}\right) \rightarrow 1$ ) are all brought about by the action of large vorticity structures residing in the outermost boundary of the turbulent flows. These findings highlight the role of large scale motions in the local transport of the passive scalar close to the TNTI outline.

So far, we have studied the dependence of the scalar concentration close to the boundary on the geometric features of the TNTI outline. It is also instructive to look at the conditionally averaged passive scalar profiles [e.g., Fig. 5(b)] with further conditioning on the different geometric features of the TNTI outline. These profiles are denoted $\left.\langle\phi\rangle_{I}\right|_{f}$, where $f$ represents the independent geometric variables as in Fig. 11. To find, say, the profiles of $\left.\langle\phi\rangle_{I}\right|_{r_{I}}$, we first identify the points along the TNTI outline that fall within a specific range of radial positions. Thereafter, the evolution of the scalar concentration along the interface-normal direction is collected and averaged, using the same procedure as in Figs. 5(b)-5(d), for that bundle of TNTI outline points. For example, the average of the instantaneous concentration profiles for interface points with $r_{I} / b_{\phi, 1 / 2}<0.5$ is shown by the black circles in Fig. 12(a), while the

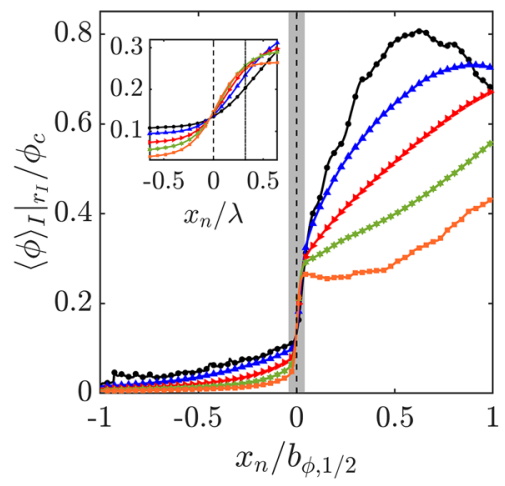

(a)

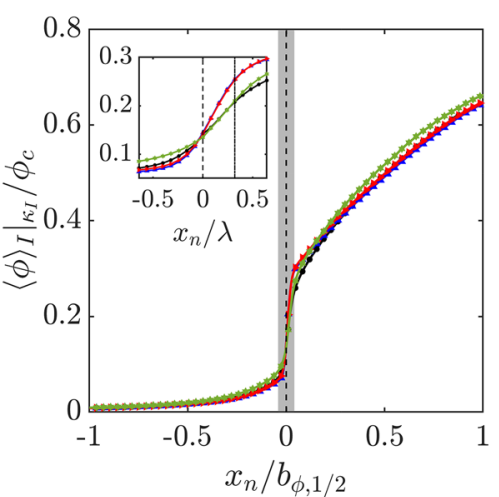

(b)

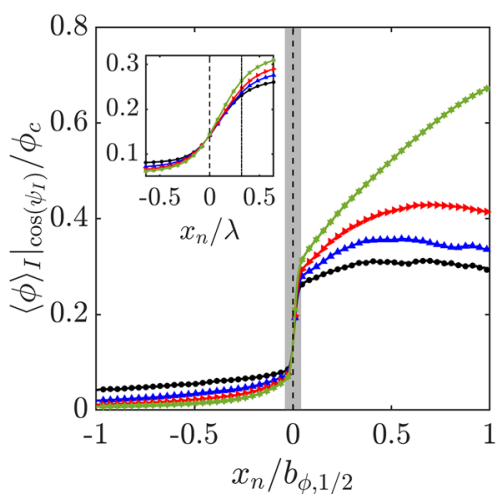

(c)

FIG. 12. (a) Conditionally averaged profiles of scalar concentration for $r_{l} / b_{\phi, 1 / 2}<0.5$ (black circles), $0.5 \leqslant r_{l} / b_{\phi, 1 / 2}<1$ (blue up-pointing triangles), $1 \leqslant r_{l} / b_{\phi, 1 / 2}<1.5$ (red right-pointing triangles), $1.5 \leqslant r_{l} / b_{\phi, 1 / 2}<2$ (green stars), and $r_{l} / b_{\phi, 1 / 2} \geqslant 2$ (orange squares). (b) Conditional profiles for $\kappa_{l} \lambda<-10$ (black circles), $-10 \leqslant \kappa_{l} \lambda<0$ (blue up-pointing triangles), $0 \leqslant \kappa_{l} \lambda<10$ (red right-pointing triangles), and $\kappa_{l} \lambda \geqslant 10$ (green stars). (c) Conditionally averaged concentration profiles for $\cos \left(\psi_{l}\right)<-0.5$ (black circles), $-0.5 \leqslant \cos \left(\psi_{l}\right)<0$ (blue up-pointing triangles), $0 \leqslant \cos \left(\psi_{l}\right)<0.5$ (red right-pointing triangles), and $\cos \left(\psi_{l}\right) \geqslant 0.5$ (green stars). The inset plots zoom in the gray region with their $x$-axis normalized by the Taylor microscale. The dashed and dashed-dotted lines represent $x_{n} / \lambda=0$ (TNTI outline) and $x_{n} / \lambda=0.32$ (investigation point), respectively. 
orange squares denote the profile for the furthest radial positions, $r_{I} / b_{\phi, 1 / 2} \geqslant 2$.

It is clear that the largest concentration values within the jet occur close to the centerline. This is confirmed in Fig. 12(a), where the boundary points closest to the centerline are associated with the largest concentration values inside the fully turbulent region. However, as we have shown earlier in Fig. 11(a), the passive scalar in the vicinity of the TNTI outline is maximized at far radial positions. This trend is consistent with the behavior of $\left.\langle\phi\rangle_{I}\right|_{r_{I}}$ within the TNTI region (the inset of Fig. 12), where we see the largest and smallest concentration values for $1.5 \leqslant r_{I} / b_{\phi, 1 / 2}<2$ and $r_{I} / b_{\phi, 1 / 2}<0.5$, respectively. Hence, the passive scalar at the TNTI outline is governed by a steeper gradient when there is lower streamwise momentum, which results in molecular diffusion acting more rapidly. By the end of the TNTI region at $x_{n} / \lambda=0.64$, the two profiles with the largest radial positions attain an approximate plateau, and the trend in the turbulent core region is already observed. Furthermore, the profiles of $\langle\phi\rangle_{I} \mid r_{I}$ with $r_{I} / b_{\phi, 1 / 2}<1$ exhibit a peak and then decrease within one scalar half-width from the TNTI outline in the turbulent region. This was expected as the interpolated flow points for these cases pass the jet centerline and approach the bottom half of the turbulent region, where the scalar concentration drops. Note that the fluctuations in the profiles of the smallest $\left(r_{I} / b_{\phi, 1 / 2}<0.5\right)$ and largest $\left(r_{I} / b_{\phi, 1 / 2} \geqslant 2\right)$ radial positions are due to an insufficient number of TNTI outline points [see Fig. 8(a)].

Figure 12(b) presents the conditionally averaged concentration profiles for different curvature values of the TNTI outline, $\left.\langle\phi\rangle_{I}\right|_{\kappa_{I}}$. Consistently with that shown in Fig. 11(b), the passive scalar at the TNTI exhibits a sharper gradient for the flatter regions of the TNTI outline $\left(-10 \leqslant \kappa_{I} \lambda<10\right)$. Deep inside the turbulent core, however, the highly curved convex surface elements are associated with a slightly larger scalar concentration. This is similar to the behavior of the passive scalar as observed in Fig. 12(a), since it is reasonable to argue that the eddy motions responsible for forming the sharp ridges of the interface can also advect the TNTI outline closer to the jet centerline. The profiles in the non-turbulent region do not differ from each other, indicating that the passive scalar field of the non-turbulent region is decorrelated from the interface curvature.

The conditional profiles of $\left.\langle\phi\rangle_{I}\right|_{\cos \left(\psi_{I}\right)}$ in Fig. 12(c) show that the averaged concentration values for the TNTI outline points with $\cos \left(\psi_{I}\right)<0.5$ reach a plateau in the turbulent core region. Since the angle between $\hat{\boldsymbol{n}}$ and $\hat{\boldsymbol{r}}$ is at least $60^{\circ}$ in these cases, the averaging path along the interface-normal direction seldom moves toward the jet centerline, which results in limited passive scalar profiles. There are also higher values of the passive scalar in the non-turbulent region for the radially contorted interface points $\left[\cos \left(\psi_{I}\right)<-0.5\right]$. The latter can be explained by the fact that the non-turbulent interpolated flow points corresponding to $\cos \left(\psi_{I}\right)<-0.5$ are always in the neighborhood of the TNTI region of another boundary point (Fig. 7). Therefore, $\left.\langle\phi\rangle_{I}\right|_{\cos \left(\psi_{1}\right)}$ remains relatively large even deep inside the non-turbulent region as $\cos \left(\psi_{I}\right) \rightarrow-1$.

At this point, it is worthwhile discussing how the choice of the investigation point $\left(x_{n} / \lambda=0.32\right)$ affects the presented results. The inset plots in Fig. 12 contain the necessary information to address this point. It is evident from Figs. 12(b) and 12(c) that choosing any other point between the TNTI outline $\left(x_{n} / \lambda=0\right)$ and the inner limit of the TNTI region $\left(x_{n} / \lambda=0.64\right)$ does not significantly change the presented results in Figs. 11(b) and 11(c), that is, for all the interfacenormal points within the TNTI region, the scalar concentration is enhanced for flatter regions of the interface and $\cos \left(\psi_{I}\right) \rightarrow 1$. This is, however, not the case for the conditioning with respect to the radial position of the TNTI outline. Further boundary points (i.e., $\left.r_{I} / b_{\phi, 1 / 2} \geqslant 1.5\right)$ have larger concentration values up to a distance of $x_{n} / \lambda \approx 0.40-0.48$ from the TNTI outline [inset plot of Fig. 12(a)], after which the trend changes to that of the turbulent core region. Thus, the choice of $x_{n} / \lambda=0.32$ was to investigate the dependence of the scalar concentration on the interfacial geometric features in the immediate vicinity of the TNTI outline, before the transition to the fully developed turbulent state.

We now consider the two-point spatial correlation of the scalar concentration to further investigate the behavior of the passive scalar near the TNTI outline. The cross correlation function, $C_{\phi \phi}\left(x_{n}, \delta\right)$, is calculated in the interface-normal coordinates, using the two points $x_{n}$ and $x_{n}+\delta$,

$$
C_{\phi \phi}\left(x_{n}, \delta\right)=\frac{\left\langle\phi^{\prime}\left(x_{n}\right) \phi^{\prime}\left(x_{n}+\delta\right)\right\rangle_{I}}{\left\langle\phi^{\prime 2}\left(x_{n}\right)\right\rangle_{I}^{1 / 2}\left\langle\phi^{\prime 2}\left(x_{n}+\delta\right)\right\rangle_{I}^{1 / 2}},
$$

where the scalar fluctuations are calculated relative to the conditional average, i.e., $\phi^{\prime}\left(x_{n}\right)=\phi\left(x_{n}\right)-\left\langle\phi\left(x_{n}\right)\right\rangle_{I}$ [see Fig. 5(c)]. Although we do not have access to the velocity field, an attempt is made to investigate the relevant scalar transport mechanisms using the conditionally averaged cross correlation profiles. Since $S c$ is high, the passive scalar is a reliable marker of the fluid particles, and to a reasonable extent, we can describe the behavior of the flow using only the scalar field. Figures 13(a)-13(c) show the cross correlation function at $x_{n} / \lambda=0.32$ with further conditioning on $r_{I}, \kappa_{I}$, and $\cos \left(\psi_{I}\right)$, respectively. Within the TNTI region, $C_{\phi \phi}(0.32 \lambda, \delta)$ decreases rapidly from a value of one at $\delta=0$, attaining a negative minimum close to the TNTI outline in the nonturbulent region. Interestingly, for all cases, $C_{\phi \phi}$ remains positive at the TNTI outline and immediately becomes negative in the nonturbulent region. The lack of correlation between $x_{n} / \lambda=0.32$ and the non-turbulent region is partly associated with the removal of all the non-turbulent flow points from $\phi_{0.32}$, as already seen in Fig. 10.

The scalar transport takes place by the advection of the fluid elements containing the scalar and also by molecular diffusion. ${ }^{25,43}$ The conditionally averaged profiles of $\left.C_{\phi \phi}\right|_{r_{I}}$ in Fig. 13(a) show that the largest scalar correlations are associated with the furthest boundary points. Molecular diffusion can even result in positive correlations between the scalar at the TNTI and that in the non-turbulent region when there is lower streamwise momentum, i.e., $r_{I} / b_{\phi, 1 / 2} \geqslant 2$ [also see Fig. 11(a)]. In contrast, the fluid parcels containing the scalar are continually advected by the strong velocity field close to the jet centerline, potentially into another $y-z$ plane further downstream. The latter suggests a lack of correlation across the scalar field, when the origin of $x_{n}$ is located on the TNTI outline points with the smallest radial positions. This is made evident in Fig. 13(a) by the negative nearly zero $\left.C_{\phi \phi}\right|_{r_{I}}$ in the turbulent region for $r_{I} / b_{\phi, 1 / 2}<0.5$.

Within this framework, we can offer an explanation for the relation between the local entrainment/detrainment process and the radial position of the TNTI outline. The fluid elements acquire the scalar concentration by the outward propagation of the TNTI outline (entrainment), whereas the inward motion of the 


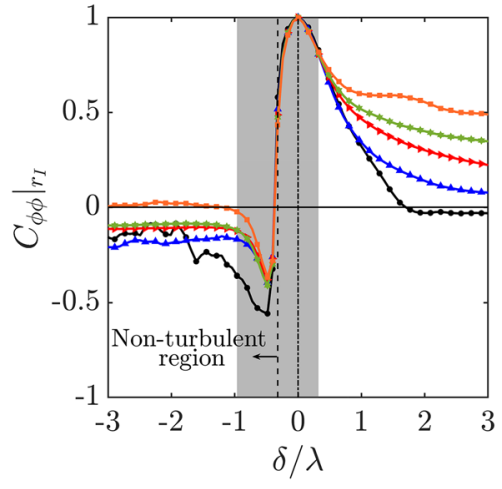

(a)

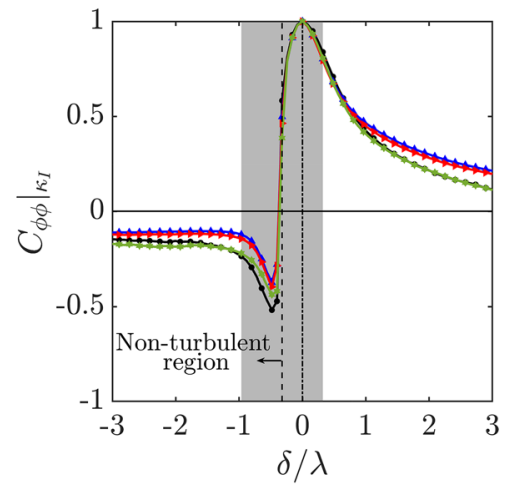

(b)

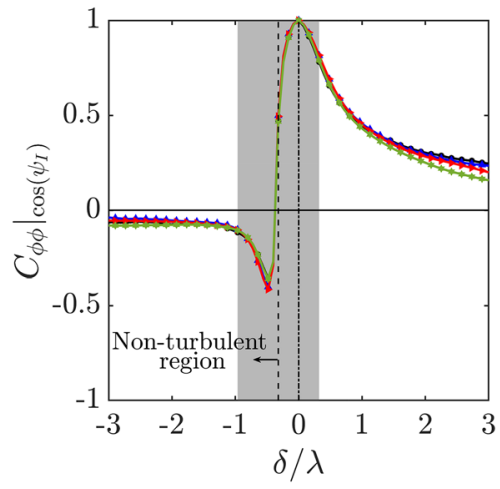

(c)

FIG. 13. Conditionally averaged cross correlation function $C_{\phi \phi}\left(x_{n}, \delta\right)$ at $x_{n} / \lambda=0.32$ with respect to the (a) radial position of the TNTI outline, $r_{l}$, (b) interface curvature, $\kappa_{l}$, and (c) cosine of the interface angle, $\cos \left(\psi_{l}\right)$. The colored markers are the same as in Fig. 12. The dashed and dashed-dotted lines represent $x_{n} / \lambda=0$ (TNTI outline) and $x_{n} / \lambda=0.32$ (investigation point), respectively.

interface toward the turbulent region causes the scalar concentration to smear out (detrainment). Recently, Mistry et al. ${ }^{21}$ found a preference for fluid detrainment at far radial distances. Formerly turbulent fluid particles are left in the non-turbulent region during detrainment, and hence, there are positive scalar correlations between the TNTI and non-turbulent regions when the radial position is away from the jet centerline [Fig. 13(a)]. Watanabe et al. ${ }^{25}$ also reported relatively large scalar correlations between the turbulent and non-turbulent regions near the trailing edge, where the interface frequently propagates toward the turbulent core. The validity of this notion can be found by directly calculating the local entrainment velocity of the scalar TNTI, $v_{n}^{\phi}=-D\left(\nabla^{2} \phi /|\nabla \phi|\right)$, where $v_{n}^{\phi} \leqslant 0$ denotes entrainment and $v_{n}^{\phi}>0$ denotes detrainment. However, lack of sufficient spatial resolution prevents us from capturing the full extent of the scalar gradient down to the Batchelor microscale, which in turn hinders the accurate calculation of $v_{n}^{\phi}$. Further study on the local entrainment velocity of the scalar TNTI at high Sc seems warranted.

The conditionally averaged profiles of $C_{\phi \phi}(0.32 \lambda, \delta)$ for the interface curvature and cosine of the interface angle are depicted in Figs. 13(b) and 13(c), respectively. Slightly weaker scalar correlations in the turbulent region can be observed near the highly curved surface elements, independently of the curvature sign, and also near the boundary points where $\hat{\boldsymbol{r}}$ and $\hat{\boldsymbol{n}}$ are preferentially aligned. The same geometric features are also associated with the strongest negative correlations in the non-turbulent region.

We may summarize the findings of Figs. 12 and 13 as follows: the evolution of the scalar concentration assembled in the interfacenormal coordinates strongly depends on the geometric features of the TNTI outline. Large eddying motions near the TNTI outline manifested in large radial positions, flat surface elements, and the alignment of the radial and normal unit vectors promote a greater scalar gradient at the TNTI. However, this description may change in the turbulent region, implying different mechanisms responsible for the transport of the scalar near the TNTI outline and inside the turbulent core. The small but positive correlation between the scalar fluctuations of the TNTI and non-turbulent regions far away from the centerline can be interpreted as the transport of the scalar to the non-turbulent region due to detrainment. In addition, the weakest scalar correlations between the TNTI and the turbulent core belong to scenarios where a larger concentration difference exists between those regions. It is also interesting that the cross correlation function displays a more significant dependence on the radial position of the interface as compared to the other two geometric variables.

\section{CONCLUSION}

The characteristics of a high-Sc passive scalar field near the turbulent/non-turbulent interface of a round momentum-driven turbulent jet have been experimentally investigated. Planar laserinduced fluorescence measurements were performed to detect the orthogonal TNTI outline at a fixed downstream position. In accordance with that reported in previous studies (e.g., Refs. 2, 44, and 61), the conditionally averaged profiles of scalar concentration, concentration rms, and concentration dissipation assembled in the interface-normal coordinates are all governed by sharp gradients across the TNTI with a thickness $\mathscr{O}(\lambda)$.

We first studied the geometric features of the TNTI outline. The PDFs of the radial position and curvature of the TNTI outline are both negatively skewed; however, the former follows a Gaussian distribution, whereas the latter presents wide tails. The time-averaged boundary has a circular shape, while concave surfaces predominate, and there is preferential alignment between the radial and normal unit vectors of the TNTI outline. Moreover, the least probable interface angle is determined to be $\psi_{I} \approx 120^{\circ}$. To complete the analysis of the interface geometry, we also showed the power-law behavior of the TNTI outline with its fractal dimension of $D_{2} \approx 1.32$ using spatial filtering.

To elucidate the dependence of the passive scalar evolution on the interface geometry, we conditioned the concentration value close to the TNTI outline on the aforementioned geometric 


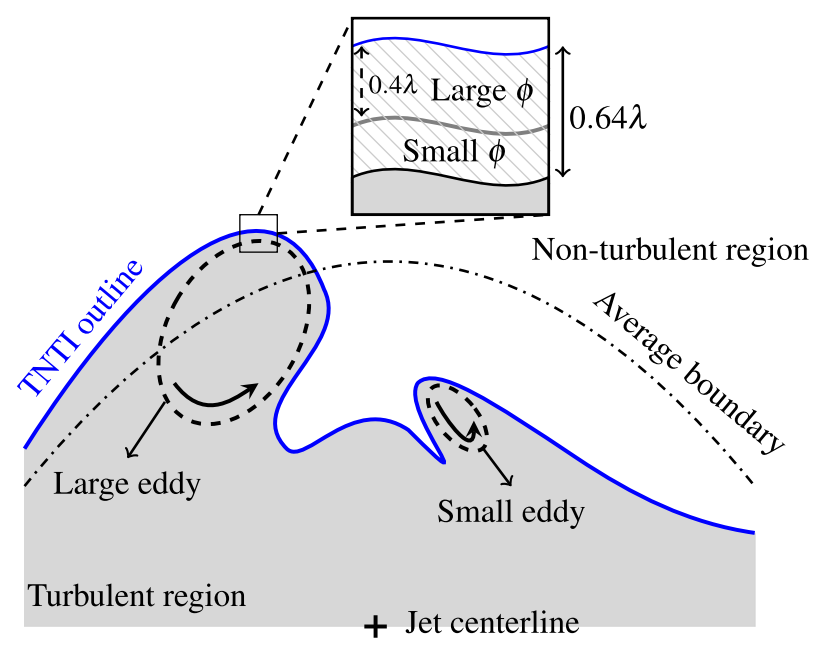

FIG. 14. Schematic of the scalar field characteristics near the TNTI outline (blue) based on the available data. The influence of different vorticity structures on the concentration value is shown. The average boundary is shown by a dashed-dotted arc. The inset is a zoomed-in view of the TNTI region (gray hatched) at far radial distances. The behavior of the TNTI closer to the jet centerline is the opposite (not shown).

variables. The results revealed an increase in the scalar concentration value in the vicinity of the TNTI outline when the interface is characterized by a moderate/large radial position, small curvature value, and small misalignment between the radial and normal unit vectors, all of which are caused by the large vorticity structures. Nonetheless, the evolution of the scalar field changes toward the inner boundary of the TNTI region, having greater concentration magnitudes along the interface-normal coordinates when the origin of $x_{n}$ is closer to the jet centerline $\left(r_{I} / b_{\phi, 1 / 2}<1.5\right)$. Highly convex surface elements also exhibit the largest concentration values deep inside the turbulent core. Furthermore, using the conditionally averaged two-point cross correlation function, we concluded that molecular diffusion becomes important far away from the centerline and can induce positive scalar correlations between the TNTI and non-turbulent regions. This may suggest local detrainment of fluid elements across the TNTI at regions characterized by low streamwise momentum, consistent with that reported in Ref. 21. A schematic picture of the aforementioned observations is illustrated in Fig. 14.

In this paper, we tried to link the coherent structures at the edge of the turbulent jet ${ }^{11,60}$ to the local scalar field via geometric features of the TNTI outline. However, higher resolution and additional information regarding the velocity field are required to further support the presented results.

\section{ACKNOWLEDGMENTS}

The authors acknowledge the anonymous referees for valuable comments. S. Gaskin acknowledges the financial support provided by the Natural Sciences and Engineering Research Council (NSERC) of Canada discovery grant (No. RGPIN 2016-04473).

\section{APPENDIX: EFFECT OF THE SPATIAL RESOLUTION ON THE CONDITIONAL STATISTICS}

Watanabe et al. ${ }^{43}$ showed that the conditionally averaged scalar profiles are less sensitive to molecular diffusion at high Sc. Here,

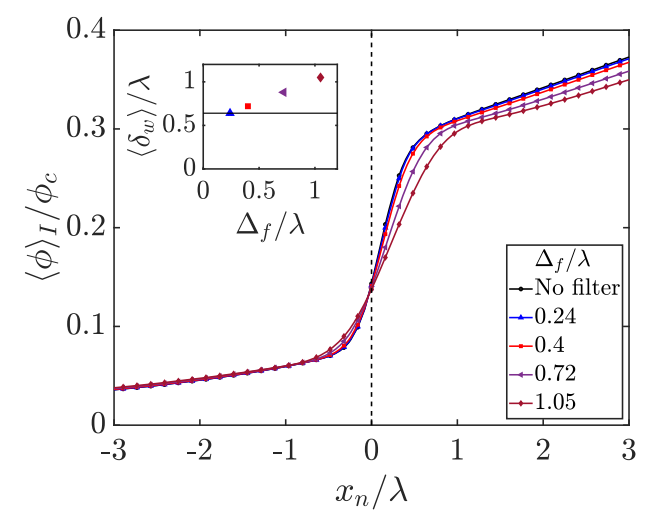

(a)

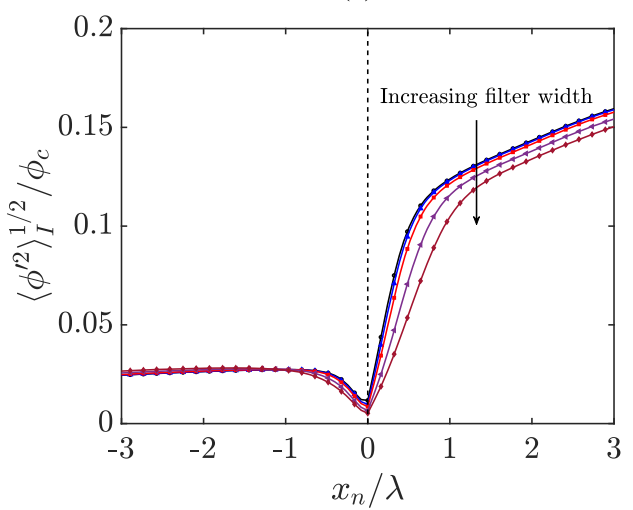

(b)

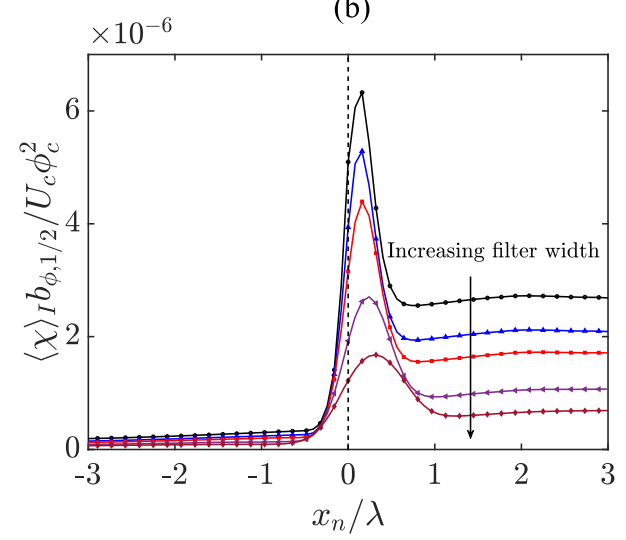

(c)

FIG. 15. Conditionally averaged profiles of (a) scalar concentration, (b) scalar concentration rms, and (c) scalar concentration dissipation as a function of various filter widths, $\Delta_{f}$. The inset plot in (a) presents the evolution of the average TNTI thickness, $\left\langle\delta_{w}\right\rangle$, against $\Delta_{f}$. The horizontal line indicates $\left\langle\delta_{w}\right\rangle$ for the original (unfiltered) concentration field. 
we investigate the effect of the spatial resolution on the conditional profiles presented in Figs. 5(b)-5(d), as well as on the thickness of the TNTI. To this end, we filter the scalar concentration fields, as explained in Sec. III A, and recalculate the conditionally averaged variables. Indeed, using a range of filter widths from $0.24 \lambda(\approx 5.7 \eta)$ to $1.05 \lambda(\approx 25 \eta)$, we were able to confirm the adequacy of the present resolution to correctly identify the TNTI thickness.

Figure 15 shows the effect of spatial filtering on the conditionally averaged profiles of scalar concentration, concentration rms, and concentration dissipation, respectively. The mean TNTI thickness normalized by the Taylor microscale, $\left\langle\delta_{w}\right\rangle / \lambda$, is calculated from the linear fits made to the conditional concentration profile (see Sec. II C) and is also shown in the inset of Fig. 15(a). The TNTI thickness is an increasing function of the filter width, i.e., a lower spatial resolution results in a thicker interfacial layer, similar to that reported by Zhang et al., ${ }^{65}$ who studied the effect of the spatial resolution on the TNTI of boundary layers. This is also consistent with the smoothed concentration profile across the TNTI at lower spatial resolutions. Interestingly, the TNTI thickness is rather insensitive to spatial filtering for the smallest filter width, $\Delta_{f}=0.24 \lambda$. This is made evident by the apparent collapse of the conditional concentration profiles, corresponding to the no filter and $\Delta_{f}=0.24 \lambda$ cases. The same observation holds true for the conditional rms profiles [Fig. 15(b)], where we see only slight differences between the no filter and $\Delta_{f}=0.24 \lambda$ cases. Thus, we conclude that the conditionally averaged concentration and concentration rms are properly captured using the present experimental resolution.

This is not the case for the conditional scalar dissipation profile since the scalar gradient quite sensitively depends on the measurement resolution. As can be seen in Fig. 15(c), with the decreasing spatial resolution, the value and width of the dissipation peak decreases and increases, respectively, consistent with the progressively smoother concentration field. Hence, the conditionally averaged scalar dissipation cannot be reliably computed at the resolution afforded by the experiment. Nonetheless, the location of the dissipation peak (i.e., $x_{n} / \lambda=0.16$ ) seems to remain preserved, at least for the first two filter widths, and we can capture the sharp jump, although it is underestimated.

Finally, we note that although the current experimental resolution $(\approx 2 \eta)$ is sufficient for calculating the conditionally averaged concentration and concentration rms profiles, molecular diffusion still strongly affects the evolution of the instantaneous scalar field within the TNTI. ${ }^{43}$ We were able to confirm this notion by evaluating the PDF of $\phi_{0.32} / \phi_{c}$, similar to Fig. 10 for each filter width (not shown).

\section{DATA AVAILABILITY}

The data that support the findings of this study are available from the corresponding author upon reasonable request.

\section{REFERENCES}

${ }^{1}$ E. Effelsberg and N. Peters, "A composite model for the conserved scalar PDF," Combust. Flame 50, 351 (1983).

${ }^{2}$ D. K. Bisset, J. C. R. Hunt, and M. M. Rogers, “The turbulent/non-turbulent interface bounding a far wake," J. Fluid Mech. 451, 383 (2002).
${ }^{3}$ J. Westerweel, C. Fukushima, J. M. Pedersen, and J. C. R. Hunt, "Mechanics of the turbulent-nonturbulent interface of a jet," Phys. Rev. Lett. 95, 174501 (2005).

${ }^{4}$ M. Holzner, A. Liberzon, N. Nikitin, W. Kinzelbach, and A. Tsinober, "Smallscale aspects of flows in proximity of the turbulent/nonturbulent interface," Phys. Fluids 19, 071702 (2007).

${ }^{5}$ C. B. da Silva and J. C. F. Pereira, "Invariants of the velocity-gradient, rate-ofstrain, and rate-of-rotation tensors across the turbulent/nonturbulent interface in jets," Phys. Fluids 20, 055101 (2008).

${ }^{6}$ S. Corrsin and A. L. Kistler, "Free-stream boundaries of turbulent flows," NACA Technical Report 1244, 1955, p. 1033.

${ }^{7}$ R. R. Taveira and C. B. da Silva, "Characteristics of the viscous superlayer in shear free turbulence and in planar turbulent jets," Phys. Fluids 26, 021702 (2014).

${ }^{8}$ R. Jahanbakhshi and C. K. Madnia, "Viscous superlayer in a reacting compressible turbulent mixing layer," J. Fluid Mech. 848, 743 (2018).

${ }^{9}$ C. B. da Silva, J. C. R. Hunt, I. Eames, and J. Westerweel, "Interfacial layers between regions of different turbulence intensity," Annu. Rev. Fluid Mech. 46, 567 (2014).

${ }^{10}$ M. van Reeuwijk and M. Holzner, "The turbulence boundary of a temporal jet," J. Fluid Mech. 739, 254 (2014).

${ }^{11}$ C. B. da Silva and R. R. Taveira, "The thickness of the turbulent/nonturbulent interface is equal to the radius of the large vorticity structures near the edge of the shear layer," Phys. Fluids 22, 121702 (2010).

${ }^{12}$ T. S. Silva, M. Zecchetto, and C. B. da Silva, “The scaling of the turbulent/nonturbulent interface at high Reynolds numbers," J. Fluid Mech. 843, 156 (2018).

${ }^{13}$ J. Westerweel, C. Fukushima, J. M. Pedersen, and J. C. R. Hunt, "Momentum and scalar transport at the turbulent/non-turbulent interface of a jet," J. Fluid Mech. 631, 199 (2009).

${ }^{14}$ M. Gampert, J. Boschung, F. Hennig, M. Gauding, and N. Peters, "The vorticity versus the scalar criterion for the detection of the turbulent/non-turbulent interface," J. Fluid Mech. 750, 578 (2014).

${ }^{15}$ K. Chauhan, J. Philip, C. M. de Silva, N. Hutchins, and I. Marusic, "The turbulent/non-turbulent interface and entrainment in a boundary layer," J. Fluid Mech. 742, 119 (2014).

${ }^{16}$ M. Holzner and B. Lüthi, "Laminar superlayer at the turbulence boundary," Phys. Rev. Lett. 106, 134503 (2011).

${ }^{17}$ M. Wolf, B. Lüthi, M. Holzner, D. Krug, W. Kinzelbach, and A. Tsinober, "Investigations on the local entrainment velocity in a turbulent jet," Phys. Fluids 24, 105110 (2012).

${ }^{18}$ J. Philip, I. Bermejo-Moreno, D. Chung, and I. Marusic, "Characteristics of the entrainment velocity in a developing wake," in International Symposium on Turbulence and Shear Flow Phenomena, TSFP-9, Melbourne, Australia, 2015.

${ }^{19}$ R. Jahanbakhshi and C. K. Madnia, "Entrainment in a compressible turbulent shear layer,” J. Fluid Mech. 797, 564 (2016).

${ }^{20}$ T. Watanabe, C. B. da Silva, K. Nagata, and Y. Sakai, "Geometrical aspects of turbulent/non-turbulent interfaces with and without mean shear," Phys. Fluids 29, 085105 (2017).

${ }^{21}$ D. Mistry, J. Philip, and J. R. Dawson, "Kinematics of local entrainment and detrainment in a turbulent jet,” J. Fluid Mech. 871, 896 (2019).

${ }^{22}$ G. Balamurugan, A. Rodda, J. Philip, and A. C. Mandal, "Characteristics of the turbulent non-turbulent interface in a spatially evolving turbulent mixing layer," J. Fluid Mech. 894, A4 (2020).

${ }^{23}$ M. Wolf, M. Holzner, B. Lüthi, D. Krug, W. Kinzelbach, and A. Tsinober, "Effects of mean shear on the local turbulent entrainment process," J. Fluid Mech. 731, 95 (2013).

${ }^{24}$ M. Wolf, B. Lüthi, M. Holzner, D. Krug, W. Kinzelbach, and A. Tsinober, "Erratum: Investigations on the local entrainment velocity in a turbulent jet [Phys. Fluids 24, 105110 (2012)]," Phys. Fluids 25, 019901 (2013).

${ }^{25}$ T. Watanabe, Y. Sakai, K. Nagata, Y. Ito, and T. Hayase, "Enstrophy and passive scalar transport near the turbulent/non-turbulent interface in a turbulent planar jet flow," Phys. Fluids 26, 105103 (2014).

${ }^{26}$ A. A. Townsend, The Structure of Turbulent Shear Flow (Cambridge University Press, 1976).

${ }^{27}$ J. Lee, H. J. Sung, and T. A. Zaki, "Signature of large-scale motions on turbulent/non-turbulent interface in boundary layers," J. Fluid Mech. 819, 165 (2017). 
${ }^{28}$ A. A. Townsend, "The mechanism of entrainment in free turbulent flows," J. Fluid Mech. 26, 689 (1966).

${ }^{29} \mathrm{G}$. L. Brown and A. Roshko, "On density effects and large structure in turbulent mixing layers,” J. Fluid Mech. 64, 775 (1974).

${ }^{30}$ J. Mathew and A. J. Basu, "Some characteristics of entrainment at a cylindrical turbulence boundary," Phys. Fluids 14, 2065 (2002).

${ }^{31}$ R. R. Taveira, J. S. Diogo, D. C. Lopes, and C. B. da Silva, "Lagrangian statistics across the turbulent-nonturbulent interface in a turbulent plane jet," Phys. Rev. E 88, 043001 (2013)

${ }^{32}$ C. B. da Silva, R. R. Taveira, and G. Borrell, "Characteristics of the turbulent/nonturbulent interface in boundary layers, jets and shear-free turbulence," J. Phys.: Conf. Ser. 506, 012015 (2014).

${ }^{33}$ J. Philip and I. Marusic, "Large-scale eddies and their role in entrainment in turbulent jets and wakes," Phys. Fluids 24, 055108 (2012).

${ }^{34}$ D. Krug, M. Holzner, B. Lüthi, M. Wolf, W. Kinzelbach, and A. Tsinober, "The turbulent/non-turbulent interface in an inclined dense gravity current," J. Fluid Mech. 765, 303 (2015).

${ }^{35}$ K. R. Sreenivasan, R. Ramshankar, and C. Meneveau, "Mixing, entrainment and fractal dimensions of surfaces in turbulent flows," Proc. R. Soc. London, Ser. A 421, 79 (1989).

${ }^{36}$ C. M. de Silva, J. Philip, K. Chauhan, C. Meneveau, and I. Marusic, "Multiscale geometry and scaling of the turbulent-nonturbulent interface in high Reynolds number boundary layers," Phys. Rev. Lett. 111, 044501 (2013).

${ }^{37}$ D. Mistry, J. Philip, J. R. Dawson, and I. Marusic, "Entrainment at multi-scales across the turbulent/non-turbulent interface in an axisymmetric jet," J. Fluid Mech. 802, 690 (2016).

${ }^{38}$ D. Mistry, J. R. Dawson, and A. R. Kerstein, "The multi-scale geometry of the near field in an axisymmetric jet," J. Fluid Mech. 838, 501 (2018).

${ }^{39}$ D. Krug, M. Holzner, I. Marusic, and M. van Reeuwijk, "Fractal scaling of the turbulence interface in gravity currents," J. Fluid Mech. 820, R3 (2017).

${ }^{40}$ Y. Zhuang, H. Tan, H. Huang, Y. Liu, and Y. Zhang, "Fractal characteristics of turbulent-non-turbulent interface in supersonic turbulent boundary layers," J. Fluid Mech. 843, R2 (2018).

${ }^{41}$ M. van Reeuwijk and J. Craske, "Energy-consistent entrainment relations for jets and plumes," J. Fluid Mech. 782, 333 (2015).

${ }^{42}$ H. Tennekes and J. L. Lumley, A First Course in Turbulence (MIT Press, 1972).

${ }^{43} \mathrm{~T}$. Watanabe, Y. Sakai, K. Nagata, Y. Ito, and T. Hayase, "Turbulent mixing of passive scalar near turbulent and non-turbulent interface in mixing layers," Phys. Fluids 27, 085109 (2015).

${ }^{44}$ T. S. Silva and C. B. da Silva, "The behaviour of the scalar gradient across the turbulent/non-turbulent interface in jets," Phys. Fluids 29, 085106 (2017).

${ }^{45} \mathrm{~T}$. Watanabe, X. Zhang, and K. Nagata, "Turbulent/non-turbulent interfaces detected in DNS of incompressible turbulent boundary layers," Phys. Fluids 30, 035102 (2018)

${ }^{46}$ C. Dopazo, J. Martin, and J. Hierro, "Local geometry of isoscalar surfaces," Phys. Rev. E 76, 056316 (2007).
${ }^{47} \mathrm{X}$. Zhang, T. Watanabe, and K. Nagata, "Passive scalar mixing near turbulent/non-turbulent interface in compressible turbulent boundary layers," Phys. Scr. 94, 044002 (2019).

${ }^{48}$ F. Hunger, M. Gauding, and C. Hasse, "On the impact of the turbulent/nonturbulent interface on differential diffusion in a turbulent jet flow," J. Fluid Mech. 802, R5 (2016).

${ }^{49}$ R. Jahanbakhshi and C. K. Madnia, "Scalar transport near the turbulent/nonturbulent interface in reacting compressible mixing layers," in Modeling and Simulation of Turbulent Mixing and Reaction, edited by D. Livescu, A. G. Nouri, F. Battaglia, and P. Givi (Springer, Singapore, 2020), p. 25.

${ }^{50}$ P. E. Dimotakis, “The mixing transition in turbulent flows," J. Fluid Mech. 409, 69 (2000).

${ }^{51}$ B. Khorsandi, "Effect of background turbulence on an axisymmetric turbulent jet," Ph.D. dissertation (McGill University, Department of Mechanical Engineering, 2011).

${ }^{52}$ A. Perez-Alvarado, "Effect of background turbulence on the scalar field of a turbulent jet," Ph.D. dissertation (McGill University, Department of Mechanical Engineering, 2016).

${ }^{53}$ H. A. Becker, H. C. Hottel, and G. C. Williams, "The nozzle-fluid concentration field of the round, turbulent, free jet," J. Fluid Mech. 30, 285 (1967).

${ }^{54}$ A. D. Birch, D. R. Brown, M. G. Dodson, and J. R. Thomas, "The turbulent concentration field of a methane jet," J. Fluid Mech. 88, 431 (1978).

${ }^{55}$ W. J. A. Dahm and P. E. Dimotakis, "Measurements of entrainment and mixing in turbulent jets," AIAA J. 25, 1216 (1987).

${ }^{56} \mathrm{P}$. N. Papanicolau and E. J. List, "Statistical and spectral properties of tracer concentration in round buoyant jets," Int. J. Heat Mass Transfer 30, 2059 (1987).

${ }^{57}$ S. K. Lele, "Compact finite difference schemes with spectral-like resolution," J. Comput. Phys. 103, 16 (1992).

${ }^{58}$ R. R. Prasad and K. R. Sreenivasan, "Scalar interfaces in digital images of turbulent flows," Exp. Fluids 7, 259 (1989).

${ }^{59}$ J. Eisma, J. Westerweel, G. Ooms, and G. E. Elsinga, "Interfaces and internal layers in a turbulent boundary layer," Phys. Fluids 27, 055103 (2015).

${ }^{60}$ R. Jahanbakhshi, N. S. Vaghefi, and C. K. Madnia, "Baroclinic vorticity generation near the turbulent/ non-turbulent interface in a compressible shear layer," Phys. Fluids 27, 105105 (2015).

${ }^{61}$ A. Attili, J. C. Cristancho, and F. Bisetti, "Statistics of the turbulent/nonturbulent interface in a spatially developing mixing layer," J. Turbul. 15, 555 (2014).

${ }^{62}$ K. A. Buch and W. J. A. Dahm, "Experimental study of the fine-scale structure of conserved scalar mixing in turbulent shear flows. Part 1. Sc > 1," J. Fluid Mech. 317, 21 (1996).

${ }^{63}$ Z. Warhaft, "Passive scalars in turbulent flows," Annu. Rev. Fluid Mech. 32, 203 (2000).

${ }^{64}$ B. B. Mandelbrot, The Fractal Geometry of Nature (W. H. Freeman and Company, 1982).

${ }^{65} \mathrm{X}$. Zhang, T. Watanabe, and K. Nagata, "Turbulent/nonturbulent interfaces in high-resolution direct numerical simulation of temporally evolving compressible turbulent boundary layers," Phys. Rev. Fluids 3, 094605 (2018). 